\title{
Real-time simulation of cable pay-out and reel-in with towed fishing gears
}

\author{
Francisco González, Amelia de la Prada, Alberto Luaces, Manuel González
}

This is a post-peer-review, pre-copyedit version of an article published in Ocean Engineering. The final authenticated version is available online at: https://doi.org/10.1016/j.oceaneng. 2017.01.002. This document is licensed under a CC-BY-NC-ND license.

\begin{abstract}
Achieving real-time simulation of fast cable pay-out and reel-in manoeuvres with towed fishing gears is a challenging task. This work presents two new simulation methods based on simplified cable models for this kind of application. First, three numerical techniques are proposed to enhance a classical spring-based cable model, increasing its computational efficiency in manoeuvres that involve reeling the cable around a winch drum. Second, the development of an efficient multibody modelling approach based on natural coordinates is reported. The performance of these methods was assessed with two realistic examples. The numerical experiments involved different values of cable axial stiffness and spatial discretization levels, since these parameters were found to have a major impact on computational efficiency. The proposed methods achieved real-time performance in the simulation of systems modelled with up to a few thousand variables. Each modelling approach has advantages and limitations that must be considered when addressing a given application.
\end{abstract}

Keywords: Underwater cable dynamics, Let-out and reel-in manoeuvres, Fishing gears, Multibody dynamics 


\section{Introduction}

Towed fishing gears such as trawls and seines are responsible for 70\% of the world fish catch [58]. These gears are complex mechanical structures mainly comprised of netting and cables. Increasing concerns about environmental impact and energy efficiency in the fishing industry are driving the development of numerical models especially suited for these kinds of assemblies, aiming at designing new gears with improved catch capability and selectivity and reduced environmental impact $[25,26,31,56]$.

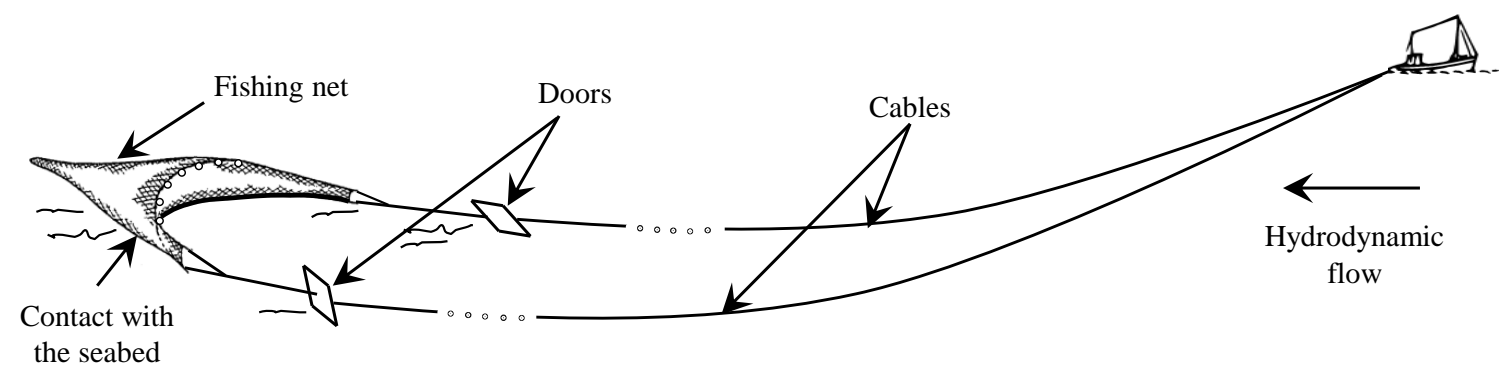

Figure 1: Components of a fishing trawl

Detailed information about the design and classification of fishing gears can be found in [38]. Fig. 1 shows a representation of a trawl, the predominant type of towed fishing gear. It consists of a flexible cone-shaped net that is pulled through the water by two long cables ( 0.5 - 3 kilometres) attached to winches on a fishing trawler. Towing speed ranges from 0.5 to 3.5 $\mathrm{m} / \mathrm{s}$. The net is made up of polygonal netting panels sewn to each other and is connected to other elements to ensure its proper deployment and buoyancy. The vertical spread of the net is provided by floats and weights placed on its upper and lower edges, respectively. The horizontal spread is generated by lateral hydrodynamic forces on the doors. Trawl doors weigh between 0.5 and 5 tons and their surface area ranges from 2 to $12 \mathrm{~m}^{2}$, depending on the net size. Midwater trawls work without contacting the seabed, while in bottom trawls the lower part of the net is in contact with the sea floor.

A fishing haul has three stages: shooting, towing, and heaving. Shooting consists of paying out the cables at constant speed, around 1-2 m/s, while the trawler sails until they achieve the desired length and the gear gets completely deployed. During towing, the winch control system regulates the cable length and tension to keep them within their admissible ranges. The cable tension during this operation can reach up to several dozen tons. State-of-the-art control systems 
also attempt to maintain the symmetry of the gear, which can be affected by ocean currents. To achieve this, they combine information from different sensors on the gear and trawler and operate the winch to let out or reel in the cables at high rates. Modern electric winches can reach reeling velocities of up to $250 \mathrm{~m} / \mathrm{min}$ in less than 1 second, starting from rest. The towing stage can last from 15 minutes to several hours. During heaving, the cables are reeled in; the control system keeps their tension under a maximum admissible value to avoid damaging the gear.

Efforts to simulate towed fishing gears have mainly focused on the calculation of the static equilibrium shape of the gear subjected to a constant water flow, although methods to deal with dynamic equilibrium can be found in the literature as well. Authors have proposed different methods to discretize the net and solve the resulting equations [28, 29, 40, 45, 47, 55], experimental procedures to measure elastic properties of netting [44, 52, 53], models for hydrodynamic forces on the gear $[6,16]$, and methods to optimize gear design $[26,48]$.

A more challenging application is the simulation of towing in order to design and evaluate new concepts of gear control [51]. This kind of co-simulation uses a dynamic model of the fishing gear as plant model inside the simulation of the control system. In some cases, these simulations are used in human-in-the-loop setups, i.e., applications that require real-time user interaction with the control system. Efficient dynamic formulations are then required to deal with the fast pay-out and reel-in motion of the cables in real-time.

Cable mechanics has been the subject of intensive studies during the last five decades. Stateof-the-art programs for the analysis of submerged cables use a spatial discretization of the continuous partial differential equations of the cable based on the finite element method [7] or finite differences [17]. These approaches include the effect of all kinds of geometric and material nonlinearities and their robustness allows one to simulate constant-length towing cables with step-sizes of seconds [17]. However, their computational overhead prevents their use in some applications that require real-time computations such as the above-mentioned human-inthe-loop simulation of gear control systems, in which fast dynamics demands the use of small integration step-sizes [23].

An approach to achieve high computational efficiency is to use simplified cable models at the expense of neglecting some geometric and material nonlinearities, such as bending or torsional stiffness and cross section reduction due to axial strain [41]. In practice, the validity of these models is determined by the characteristics of the application under study. In fishing assemblies, 
the range of cable tensions and moderate torsion and curvature radius make these simplifications acceptable [50]. Often, simplified models discretize the cable as a sequence of segments connected by joints that allow them to rotate with respect to each other; bending stiffness can be modelled with torsion springs between bars [15]. These segments can be flexible or rigid along their longitudinal axis, or a combination of both [14]. Linear spring models [29, 48], are instances of the first kind; structural damping can be included in these flexible elements as well $[8,59]$. These models have been demonstrated to work well in the dynamic simulation of towed gears, and they show good agreement with experimental results [50, 57]. If the cable is discretized as a chain of rigid links, then multibody formulations can be employed to solve the dynamics of the resulting model. This method neglects the axial flexibility of the cable, which can be a valid assumption for stiff cables. The rope model described in [15] is a 2D example of this second approach; only a few publications exist that use 3D multibody modelling in practical marine applications [24], especially in the context of towed fishing gears [34].

Even with simplified cable models, real-time simulation of let-out and reel-in operations remains an exacting task. The quick motion of the system, the changes in the free length of the cable, and their effect on cable tension have to be considered and correctly dealt with. In practice, carrying out the integration with step-sizes in the range of milliseconds is mandatory. Moreover, the accurate modelling of cable behaviour, e.g., determining the contact region between the cable and the seabed, demands the use of discretizations with a relatively large number of elements, ranging from one hundred to a few thousands. Numerical methods able to perform real-time integration of the resulting dynamics equations while keeping the simulation stable have not been reported yet in the literature.

The present paper puts forward efficient computational methods for the real-time simulation of manoeuvres with submerged cables and fishing gears. The main scientific contributions of this work can be summarized as follows. First, it introduces three numerical improvement approaches to enhance the performance of classical spring-based cable models during reel-in and reel-out manoeuvres. These alleviate the time-scale reduction caused by the shortening of cable elements introduced when changes in cable length are represented with variable-size segments. Second, it reports a new multibody model of the cable, based on natural coordinates, which constitutes an efficient way to formulate the system dynamics, alternative to using spring models. The system dynamics equations were solved making use of augmented Lagrangian and Hamiltonian formulations, whose application to real-time cable dynamics had not been reported yet, and 
integrated with simple time-stepping routines. Third, the efficiency of the multibody methodology in the simulation of reel-in and reel-out motion was further improved via an accelerationlevel penalization of the dynamics of the wound cable segments. The use of these techniques made it possible to achieve real-time simulation of let-out and reel-in manoeuvres with towed fishing gears, using cable discretizations comprising up to one thousand segments.

\section{System modelling}

Two alternative approaches to cable modelling are introduced in this section. The first one is a linear spring model and the second uses a multibody discretization.

\subsection{Linear spring model of the cable}

In the context of fishing operations, cables are traditionally modelled as a series of point masses connected by linear spring elements $[29,57,50]$ as shown in Fig. 2 . This approach assumes that: (a) the effect of the bending stiffness of the cable is negligible compared to its axial stiffness; (b) the contribution of the rotational inertia of the cable to the dynamics is not significant; and (c) the structural damping of the cable is less relevant than the one introduced by hydrodynamic forces. These assumptions are valid in the context of towed fishing gears, where cable rotation is negligible and the radii of curvature are large [49].

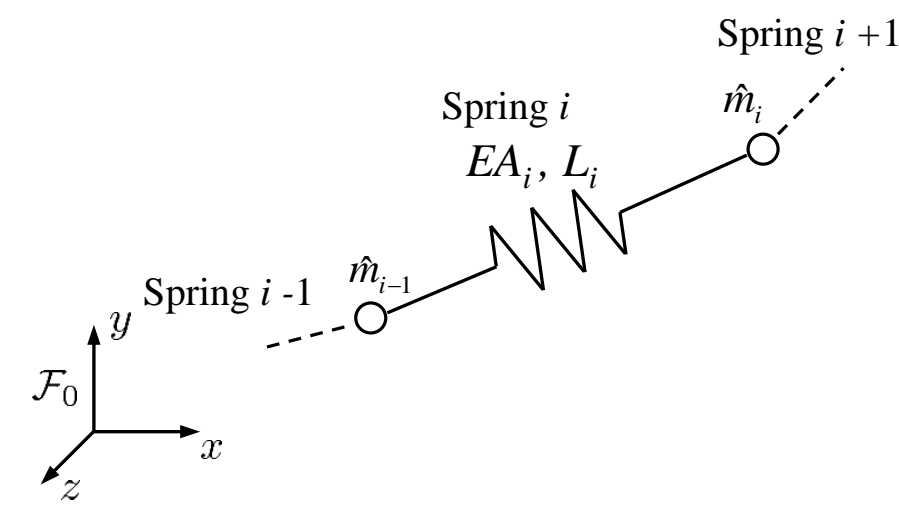

Figure 2: Discretization of a cable into a chain of mass-spring elements 
A cable of length $L$ and total mass $m$ can be discretized into $N$ springs and $N$ point masses. Fig. 2 shows the spring $i$ connecting masses $\hat{m}_{i-1}$ and $\hat{m}_{i}$, whose coordinates $\left(x_{i-1}, y_{i-1}, z_{i-1}\right)$ and $\left(x_{i}, y_{i}, z_{i}\right)$ are expressed in the global reference frame $\mathcal{F}_{0}$. The mass $\hat{m}_{i}$ is calculated as half the mass of the cable between points $i-1$ and $i+1$. The coordinates of all the point masses define the $3 N \times 1$ array of positions $\mathbf{q}=\left[x_{1}, y_{1}, z_{1}, \ldots, x_{N}, y_{N}, z_{N}\right]^{\mathrm{T}}$. The cable dynamics equations are given by

$$
\widehat{\mathbf{M}} \ddot{\mathbf{q}}=\mathbf{f}_{e}+\mathbf{f}
$$

where $\widehat{\mathbf{M}}$ is the $3 N \times 3 N$ diagonal mass matrix, $\mathbf{f}_{e}$ represents the elastic forces introduced by the linear springs, and $\mathbf{f}$ is the vector of other generalized applied forces that act on the point masses, which can be decomposed as

$$
\mathbf{f}=\mathbf{f}_{d}+\mathbf{f}_{g}+\mathbf{f}_{f}
$$

where $\mathbf{f}_{d}$ contains the hydrodynamic forces, $\mathbf{f}_{g}$ includes the weight and buoyancy, and $\mathbf{f}_{f}$ is the force due to contact with the seabed. These terms are obtained after assembling the forces that act on each element of the discretized cable, denoted by the $3 \times 1$ arrays $\widetilde{\mathbf{f}}_{e}, \widetilde{\mathbf{f}}_{d}, \widetilde{\mathbf{f}}_{g}$, and $\widetilde{\mathbf{f}}_{f}$. Terms $\widetilde{\mathbf{f}}_{d}, \widetilde{\mathbf{f}}_{g}$, and $\widetilde{\mathbf{f}}_{f}$ are common to the linear-spring and multibody models, and their expressions are provided in Section 2.5. Conversely, term $\widetilde{\mathbf{f}}_{e}$ belongs exclusively to the linear-spring model. The magnitude of the force introduced in the system by each spring, $\widetilde{f}_{e}$, can be evaluated as

$$
\tilde{f}_{e}=E A \frac{s_{i}-L_{i}}{L_{i}}
$$

where $s_{i}$ is the length of the spring, i. e., the distance between point masses $\hat{m}_{i-1}$ and $\hat{m}_{i}$, and $L_{i}$ its natural length. $E A$ denotes the cable axial stiffness per unit length, which typically ranges from $10^{5} \mathrm{~N}$ to $10^{8} \mathrm{~N}[35,36]$.

\subsection{Multibody model of the cable}

The cable can also be modelled as a multibody system composed of a chain of rigid segments, an approach that has been used several times in the literature, e.g., [24, 54, 34]. Several coordinate choices can be made in this case, and the selection has an impact on simulation efficiency and stability. In this work, natural coordinates [22] were used to represent the cable and the other 
elements that compose the fishing gear. This modelling approach describes a mechanical system using the coordinates in the global reference frame $\mathcal{F}_{0}$ of a set of representative points and vectors fixed on the system bodies.

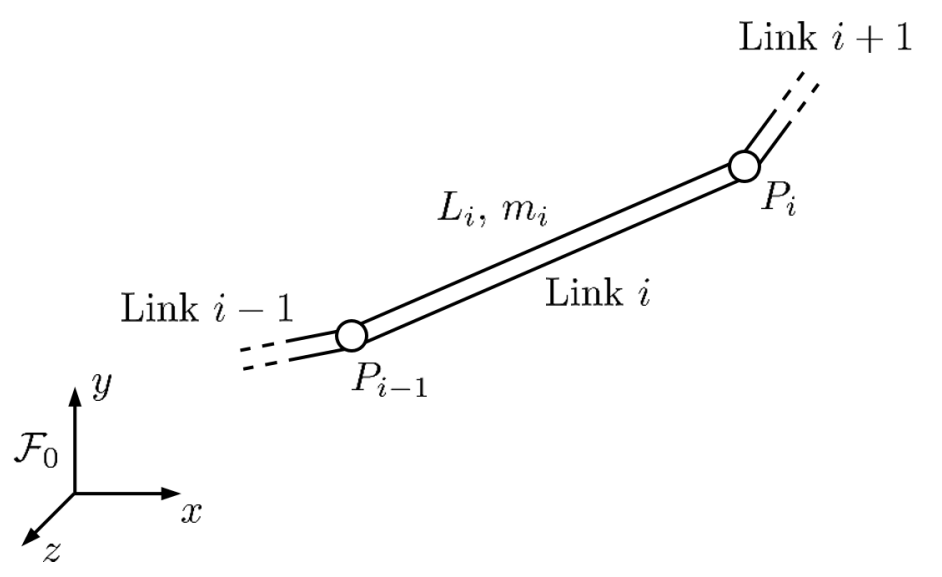

Figure 3: Discretization of a cable into an $N$-rigid-link chain

The cable is discretized into a chain of $N$ rigid links of length $L_{i}$ and mass $m_{i}$ as shown in Fig. 3. Link $i$ is connected to the previous and the next ones in the chain through ideal and frictionless spherical joints located at its extreme points $P_{i-1}$ and $P_{i}$. These points are defined by their global coordinates $\left(x_{i-1}, y_{i-1}, z_{i-1}\right)$ and $\left(x_{i}, y_{i}, z_{i}\right)$, respectively. A constant distance constraint

$$
\Phi_{i}=\left(x_{i}-x_{i-1}\right)^{2}+\left(y_{i}-y_{i-1}\right)^{2}+\left(z_{i}-z_{i-1}\right)^{2}-L_{i}^{2}=0
$$

is used to enforce the rigid-body behaviour of the link. With this modelling, the cable segments can rotate freely about their longitudinal axes.

Array q defined in Section 2.1 contains the coordinates of the $N$ points $P_{i}$. Similarly, the $N$ constraint equations $\Phi_{i}$ can be expressed as $\boldsymbol{\Phi}=\left[\Phi_{1}, \ldots, \Phi_{N}\right]^{\mathrm{T}}=\mathbf{0}$. The cable dynamics equations can then be formulated as a system of $3 N+N$ Differential Algebraic Equations (DAEs) in the form

$$
\begin{aligned}
& \mathbf{M} \ddot{\mathbf{q}}=\mathbf{f}+\mathbf{f}_{c} \\
& \mathbf{\Phi}=\mathbf{0}
\end{aligned}
$$

where $\mathbf{M}$ is the $3 N \times 3 N$ block diagonal mass matrix and $\mathbf{f}$ and $\mathbf{f}_{c}$ are the applied forces and constraint reactions, respectively. It must be noted that $\mathbf{M}$ includes the rotational inertia of the 
cable and therefore is not the same as $\widehat{\mathrm{M}}$.

Modelling the system with natural coordinates has significant advantages compared to other coordinate choices used in multibody cable models described in the literature [15, 24, 34]. In the first place, the Coriolis and velocity-dependent forces term is absent from the dynamics equations and the mass matrix $\mathbf{M}$ is constant. Moreover, it is not necessary to explicitly parametrize the rotation of the bodies of the dynamical system, i. e., additional rotation variables such as Euler angles or Euler-Rodrigues parameters are not required to describe the motion, thus avoiding well-known problems like singularities or gimbal locks [60]. Finally, the generalized coordinates $\mathbf{q}$ are the same ones used in linear spring models, which enables the easy integration of the multibody description with previously existing software for cable dynamics.

\subsection{Modelling of let-out and reel-in operations}

The dynamics of a cable during reel-in and reel-out around a winch drum were studied in [27] and [32] with inextensible cable models, based on equations particularized for a certain geometry. Even with these simplifications, the resulting dynamics equations were complex and not suitable for real-time simulation. However, the reel-in and reel-out motion of the cable around the winch drum does not need to be modelled in detail when simulating the motion of fishing gears because the winch diameter, less than $2 \mathrm{~m}$, is two or three orders of magnitude smaller than the free length of the cable. Accordingly, the winch can be modelled as a point. This enables the representation of cable reel-in and reel-out operations with variable-length cables $[24,30,34]$. This approach was followed in this work as well.

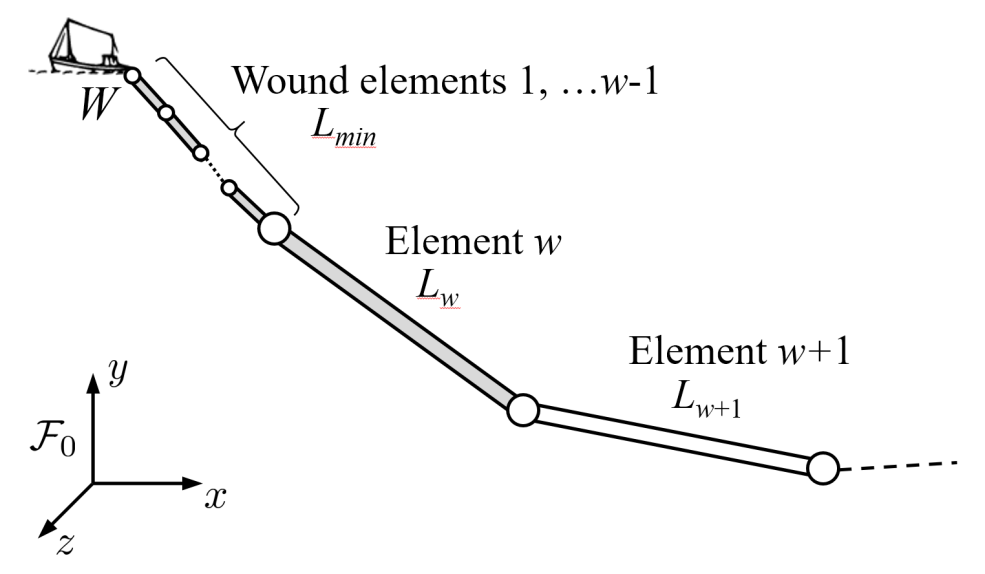

Figure 4: Modelling of reel-in operations as element retraction 
Figure 4 illustrates the way in which the length of the free cable is reduced to model a reel-in operation. The winch is located at point $W$. Let us assume that initially the cable is completely deployed. When the reel-in process begins, the length $L_{1}$ of the element closest to the winch is decreased until it reaches a minimum limit, $L_{\text {min }}$, which will be discussed in detail in Section 2.4. When this happens, the next element in the chain begins to be wound. In the following, the element that undergoes reel-in is denoted with index $w$; cable elements with indexes $i<w$ are already fully retracted and their lengths are $L_{m i n}$, as shown in Fig. 4. A reverse procedure is followed in the let-out operation.

When the spring model in Section 2.1 is used, modifying $L_{w}$ is equivalent to changing the natural length of spring $w$. If the modelling is carried out with the multibody approach in Section 2.2, the kinematic constraint in Eq. (4) can be modified with the introduction of a timevarying element length $L_{w}(t)$ to represent the shortening or extension of element $w$. Equation (4) thus becomes a rheonomic constraint. Introducing a time-dependent cable-length in the configuration-level expression of the kinematic constraints means that terms $\boldsymbol{\Phi}_{t}=\partial \boldsymbol{\Phi} / \partial t$ and $\dot{\mathbf{\Phi}}_{t}=d(\partial \boldsymbol{\Phi} / \partial t) / d t$ need be appended to their velocity-level and acceleration-level counterparts [34]

$$
\begin{aligned}
\frac{\partial \Phi_{w}}{\partial t} & =-2 L_{w} \dot{L}_{w} \\
\frac{\partial \dot{\Phi}_{w}}{\partial t} & =-2\left(\dot{L}_{w}^{2}+L_{w} \ddot{L}_{w}\right)
\end{aligned}
$$

\subsection{Numerical improvement}

Modelling reel-in and reel-out operations as changes in cable length can modify dramatically the time scale of the problem. A simple way to represent reel-in with the spring model would be considering that the cable dynamics in Eq. (1) describes only the behaviour of those cable segments that are not wound around the winch drum. This way, applying a length reduction to cable segment $w$ means that a portion of it leaves the system described by Eq. (1). Consequently, the mass of this element, $m_{w}$, must be decreased together with its natural length $L_{w}$ while its effective stiffness, $k_{w}=E A / L_{w}$, increases during the reel-in process. As a consequence, the element dynamics becomes faster, and smaller step-sizes are required to carry out the numerical 
integration of the motion, which results in a considerable computational overhead. Setting a minimum value $L_{\min }$ for the length that cable elements can reach partially alleviates these numerical problems but introduces an error in positions due to the residual lengths of the retracted cable elements.

Alternative solutions can be formulated considering that Eq. (1) represents the dynamics of all the cable elements, including those wound around the winch drum. As mentioned before, the winch can be modelled as a single point $W$, with which the lumped masses of the elements with indexes $i<w$ should be coincident during motion. These wound cable segments should not contribute to the motion of the rest of the cable, which is described only by elements with $i \geq w$. It is possible to modify the physical properties of the wound elements to model this, improving at the same time the dynamic behaviour of the simulation without affecting negatively the results. Three approaches are proposed here to avoid the introduction of artificial high frequencies in the cable dynamics during reeling-in.

The first method consists in softening the spring that is being retracted. Instead of using Eq. (3), the elastic forces exerted by this spring can be calculated as

$$
\widetilde{f}_{e}=E A \frac{s_{w}-L_{w}}{L_{w 0}}
$$

where $L_{w 0}$ is a constant value, which can be set to match the length of an unwound cable segment. If Eq. (7) is used, then the stiffness of cable segment $w, k_{w}=E A / L_{w 0}$, remains constant in spite of the variation in its natural length $L_{w}$. On the other hand, the compliance per unit length of the whole cable is increased; this will affect the tension in simulations that involve soft cables or dynamic loads. The effect of softening on cable tension will be analysed in Section 5.3.

The second strategy aims at keeping constant the natural frequency of the element in the axial direction, given by $\omega_{w}^{a x i a l}=\sqrt{k_{w} / m_{w}}$. This is done by adjusting the element mass $m_{w}$ to balance the increment of stiffness. The effect of this arrangement will also be discussed in Section 5.3.

Finally, the third strategy maintains the natural frequency $\omega_{w}^{\text {axial }}$ by keeping constant both the stiffness $k_{w}$ and the mass $m_{w}$ of cable element $w$.

Table 1 summarizes these three strategies, comparing their effects on element properties 
to those of the original unimproved model. It must be noted that methods 1 and 3 make the resultant cable more compliant than the original one.

Table 1: Effect of numerical improvement approaches for the linear spring model on the physical properties of the cable segment $w$ that is undergoing reel-in

\begin{tabular}{lcccc}
\hline Method & $\widetilde{f}_{e}$ & $m_{w}$ & $k_{w}$ & $\omega_{w}^{\text {axial }}$ \\
\hline 0 - No improvement & Eq. (3) & $\downarrow$ & $\uparrow$ & $\uparrow \uparrow$ \\
1 - Softening & Eq. (7) & $\downarrow$ & $=$ & $\uparrow$ \\
2 - Mass adjustment & Eq. (3) & $\uparrow$ & $\uparrow$ & $=$ \\
3 - Softening, fixed $m_{w}$ & Eq. (7) & $=$ & $=$ & $=$ \\
\hline
\end{tabular}

Methods No. 0 and No. 2 use Eq. (3) to evaluate the elastic forces and therefore require setting a minimum value of $L_{\min }$ to prevent its denominator from becoming zero. In this research, numerical experiments led to the selection of $L_{\text {min }}=0.03 \mathrm{~m}$ to keep the stability of the simulations. On the contrary, methods No. 1 and No. 3 dispense with $L_{\min }$ as they use Eq. (7) instead, hence removing the residual lengths of the retracted cable segments, which can be set to zero.

In the multibody model in Section 2.2 the cable elements behave as rigid bodies along their longitudinal axes and axial dynamics does not play a role in the stability of the numerical integration process. However, reducing the mass and length of an element still affects its rigid-body dynamics and demands a decrease in the integration step-size to keep the simulation stable. A possible workaround is penalizing the dynamics of the elements with indexes $i \leq w$ at the acceleration level, to remove high-frequency components from their motion and bring it closer to the ideal case, in which the point masses $m_{i}$ are coincident with the winch. This modification is equivalent to introducing an additional term in the dynamics equations (5a)

$$
(\mathbf{M}+\mathbf{P}) \ddot{\mathbf{q}}=\mathbf{f}+\mathbf{f}_{c}
$$

where $\mathbf{P}$ is a diagonal matrix whose entries are 0 for those elements with $i>w$, and a penalty factor $p_{\alpha}$ for $i<w$. The value of $p_{\alpha}$ is gradually increased or decreased for element $w$ during the reel-in or reel-out process. This method does not modify the global tension of the cable predicted by the multibody model. 


\subsection{External actions on the system}

The submerged cable is subjected to the action of hydrodynamic forces caused by the velocity difference between the cable itself and the surrounding fluid. Traditionally, in simulation of fishing nets, fluid dynamics is modelled through Morison equations particularized for a uniform current flow [33, 48, 57], discarding fluid-structure interaction. According to these expressions, the hydrodynamic forces introduce drag along the normal and tangent directions on each cable segment. These forces can be evaluated as a function of the cable element length $L_{i}$ and diameter $D$ as [43]

$$
\widetilde{\mathbf{f}}_{d n}^{\text {cable }}=\frac{1}{2} \rho C_{d} L_{i} D\left|\mathbf{v}_{n}\right| \mathbf{v}_{n}
$$

for the normal direction and

$$
\tilde{\mathbf{f}}_{d t}^{c a b l e}=\frac{1}{2} \rho C_{f} L_{i} D\left|\mathbf{v}_{t}\right| \mathbf{v}_{t}
$$

for the tangential one, where $\rho$ is the fluid density, $C_{f}$ and $C_{d}$ are friction and drag coefficients, and $\mathbf{v}_{t}$ and $\mathbf{v}_{n}$ are the tangential and normal components of the fluid velocity relative to the cable segment. In this work, the inertial term of Morison equations due to the fluid dynamics is considered to be negligible compared to the drag terms.

The contact with the seabed was modelled applying a normal contact force $\widetilde{f}_{c n}$ to each node $i$ in contact with the sea bottom. The magnitude of this force is proportional to the indentation $y_{i}-h_{s}$

$$
\widetilde{f}_{c n}=-k_{s}\left(y_{i}-h_{s}\right)
$$

where $h_{s}$ is the seabed depth. A tangential force $\widetilde{f}_{c t}$, approximated by the dynamic friction expression

$$
\widetilde{f}_{c t}=\mu_{s} \widetilde{f}_{c n}
$$

was also applied to these elements. The seabed stiffness $k_{s}$ and the friction coefficient $\mu_{s}$ depend on the characteristics of the seafloor and the cable nature. 


\section{Computational methods}

The choice of cable model determines the characteristics of the computational methods that can be used in the forward-dynamics simulation of the system motion. If the linear-spring model described in Section 2.1 is selected, the system dynamics is expressed by a system of Ordinary Differential Equations (ODEs). The multibody model in Section 2.2, on the other hand, leads to a system of DAEs. Fixed-step integrator schemes have been selected, since the computational methods are intended to be used in real-time applications.

\subsection{Numerical methods for the spring model}

Equation (1) represents a system of $3 N$ ODEs in which the second derivatives with respect to time of the node coordinates, $\ddot{\mathbf{q}}$, are the only unknowns. This system can be directly solved to obtain $\ddot{\mathbf{q}}$, and these accelerations subsequently integrated. In this work, the implicit, single-step Newmark family of integrators [39] was selected to this end,

$$
\begin{aligned}
& \mathbf{q}_{k+1}=\mathbf{q}_{k}+\Delta t \dot{\mathbf{q}}_{k}+\frac{\Delta t^{2}}{2}\left((1-2 \beta) \ddot{\mathbf{q}}_{k}+2 \beta \ddot{\mathbf{q}}_{k+1}\right) \\
& \dot{\mathbf{q}}_{k+1}=\dot{\mathbf{q}}_{k}+\Delta t\left((1-\gamma) \ddot{\mathbf{q}}_{k}+\gamma \ddot{\mathbf{q}}_{k+1}\right)
\end{aligned}
$$

where $\gamma$ and $\beta$ are scalar dimensionless parameters, $\Delta t$ is the integration step-size, and subscript $k$ refers to the integration time-step. If $\gamma=1 / 2$ and $\beta=1 / 4$, then Eq. (13) becomes the wellknown trapezoidal rule method. This integrator was used in a fixed point iteration scheme in the simulation of the linear spring cable model.

\subsection{Numerical methods for the multibody model}

Modelling the cable as a multibody system results in the dynamics expressed in the form of a system of DAEs like the one in Eqs. (5). A large number of formulations to deal with such a system have been proposed in the literature, e.g. [1, 22]. Selecting the right formulation for a given problem is critical for efficiency. For the simulation of the cable model described in Section 2.2, we have selected global formulations based on the augmented Lagrangian method, which are easy to implement and have shown good performance when dealing with moderate-size 
problems [12].

Following an augmented Lagrangian approach, it is possible to express the unknown constraint reactions as $\mathbf{f}_{c}=-\boldsymbol{\Phi}_{\mathbf{q}}^{\mathrm{T}} \boldsymbol{\lambda}$, where $\boldsymbol{\Phi}_{\mathbf{q}}=\partial \mathbf{\Phi} / \partial \mathbf{q}$ is the $N \times 3 N$ Jacobian matrix of the constraints and $\boldsymbol{\lambda}$ is a set of $N$ Lagrange multipliers. The augmented Lagrangian algorithms described in [5] and [4] follow this approach and, additionally, use a stabilized penalty method to remove the violation of kinematic constraints. An iterative algorithm is thus obtained

$$
\begin{aligned}
& \left(\mathbf{M}+\boldsymbol{\Phi}_{\mathbf{q}}^{\mathrm{T}} \alpha \boldsymbol{\Phi}_{\mathbf{q}}\right) \ddot{\mathbf{q}}+\boldsymbol{\Phi}_{\mathbf{q}}^{\mathrm{T}} \boldsymbol{\lambda}=\mathbf{f}-\boldsymbol{\Phi}_{\mathbf{q}}^{\mathrm{T}} \alpha\left(\dot{\boldsymbol{\Phi}}_{\mathbf{q}} \dot{\mathbf{q}}+\dot{\boldsymbol{\Phi}}_{t}+2 \xi \omega \dot{\boldsymbol{\Phi}}+\omega^{2} \boldsymbol{\Phi}\right) \\
& \boldsymbol{\lambda}_{j+1}=\boldsymbol{\lambda}_{j}+\alpha\left(\ddot{\mathbf{\Phi}}+2 \xi \omega \dot{\boldsymbol{\Phi}}+\omega^{2} \boldsymbol{\Phi}\right)
\end{aligned}
$$

where subscript $j$ stands for the iteration number. Term $\alpha$ is a scalar penalty factor, and coefficients $\xi$ and $\omega$ play a role similar to Baumgarte's stabilization parameters [2]. These parameters must be adjusted for optimal performance in each simulation.

It is also possible to develop augmented Lagrangian algorithms starting from Hamilton's canonical equations

$$
\dot{\mathbf{q}}=\frac{\partial H}{\partial \mathbf{p}} ; \quad-\dot{\mathbf{p}}=\frac{\partial H}{\partial \mathbf{q}}-\mathbf{f}_{n c}+\boldsymbol{\Phi}_{\mathbf{q}}^{\mathrm{T}} \boldsymbol{\lambda}
$$

where $\mathbf{f}_{n c}$ are the non-conservative forces acting on the system, $H$ is the Hamiltonian, and $\mathbf{p}$ is the $3 N \times 1$ canonical momenta term. Such methods were proposed for multibody dynamics applications in [3] following a similar procedure to that used to develop the algorithm described by Eqs. (14). The generalized velocities are obtained from

$$
\left(\mathbf{M}+\boldsymbol{\Phi}_{\mathbf{q}}^{\mathrm{T}} \alpha \boldsymbol{\Phi}_{\mathbf{q}}\right) \dot{\mathbf{q}}=\mathbf{p}-\boldsymbol{\Phi}_{\mathbf{q}}^{\mathrm{T}} \alpha\left(\boldsymbol{\Phi}_{t}+2 \xi \omega \mathbf{\Phi}+\omega^{2} \int_{t_{0}}^{t} \boldsymbol{\Phi} d t\right)-\boldsymbol{\Phi}_{\mathbf{q}}^{\mathrm{T}} \boldsymbol{\sigma}
$$

where $t_{0}$ is the initial time and $\boldsymbol{\sigma}$ are the formulation multipliers, which verify $\dot{\boldsymbol{\sigma}}=\boldsymbol{\lambda}$. The time derivatives of the canonical momenta are evaluated as

$$
\dot{\mathbf{p}}=\mathbf{f}+\dot{\boldsymbol{\Phi}}_{\mathbf{q}}^{\mathrm{T}} \alpha\left(\dot{\boldsymbol{\Phi}}+2 \xi \omega \boldsymbol{\Phi}+\omega^{2} \int_{t_{0}}^{t} \mathbf{\Phi} d t\right)+\dot{\boldsymbol{\Phi}}_{\mathbf{q}}^{\mathrm{T}} \boldsymbol{\sigma}
$$

and the multipliers $\sigma$ are updated iteratively

$$
\boldsymbol{\sigma}_{j+1}=\boldsymbol{\sigma}_{j}+\alpha\left(\dot{\boldsymbol{\Phi}}+2 \xi \omega \mathbf{\Phi}+\omega^{2} \int_{t_{0}}^{t} \mathbf{\Phi} d t\right)
$$


Two numerical integration formulas were combined with the multibody dynamics formulations. The first one was the Newmark method in Eq. (13). The second was the explicit, singlestep forward Euler integration formula. This method has shown good performance and energyconserving properties when combined with the augmented Hamiltonian algorithm described by Eqs. (16)-(18) [18]. The first order expression of the integrator used with Hamiltonian methods is

$$
\mathbf{y}_{k+1}=\mathbf{y}_{k}+\Delta t \dot{\mathbf{y}}_{k}
$$

where $\mathbf{y}=\left[\mathbf{q}^{\mathrm{T}} \mathbf{p}^{\mathrm{T}}\right]^{\mathrm{T}}$. The second order expression for the Lagrangian algorithm in Eq. (14) is

$$
\begin{aligned}
& \mathbf{q}_{k+1}=\mathbf{q}_{k}+\Delta t \dot{\mathbf{q}}_{k+1} \\
& \dot{\mathbf{q}}_{k+1}=\dot{\mathbf{q}}_{k}+\Delta t \ddot{\mathbf{q}}_{k}
\end{aligned}
$$

The Newmark formulas were combined with the augmented Lagrangian dynamics equations (14) in two ways. First, fixed point iteration with the system accelerations as primary integration variables was used. A second alternative was tested as well, which consisted of selecting the generalized coordinates as primary variables instead to obtain an index-3 algorithm. This required the introduction of the integrator formulas in the dynamics equations and the establishment of the dynamic equilibrium at time-step $k+1$. The dynamics equations thus became a system of nonlinear equations in the form

$$
\mathbf{g}(\mathbf{q}, \dot{\mathbf{q}})=\mathbf{0}
$$

which was solved by means of a Newton-Raphson iteration scheme. The stability of the time integration was ensured projecting the system velocities and accelerations onto the constraint manifold defined by the kinematic constraints $\mathbf{\Phi}=\mathbf{0}$. The resulting equations are described in detail in [10] and [11].

After a preliminary benchmarking stage, four multibody formalisms that showed the best efficiency and accuracy properties were selected and evaluated. The augmented Lagrangian method in Eqs. (14) was combined with the trapezoidal rule variant of Eq. (13) and the forward Euler expression in Eq. (20); the resulting algorithms were labelled "AL+TR" and "AL+FWE", respectively. The augmented Hamiltonian method in Eqs. (16) - (18) was integrated using the forward 
Euler formula in Eq. (19); this combination was labelled "AH+FWE". The last algorithm was the index-3 augmented Lagrangian method with velocity and acceleration projections, "ALi3p", represented by Eq. (21). These methods are summarized in Table 2.

Table 2: Summary of multibody methods used in the simulations

\begin{tabular}{lcccc}
\hline Method & Formulation & Integrator & Iteration scheme & Equations \\
\hline AL + TR & Aug. Lagrangian & Trapezoidal rule & Fixed point & $(13),(14)$ \\
AL + FWE & Aug. Lagrangian & Forward Euler & None & $(14),(20)$ \\
AH + FWE & Aug. Hamiltonian & Forward Euler & None & $(16)-(18),(19)$ \\
ALi3p & Aug. Lagrangian & Trapezoidal rule & Newton-Raphson & $(21)$ \\
\hline
\end{tabular}

It must be noted that some criterion must be defined to stop the iteration algorithm when an implicit integrator like the trapezoidal rule is used, in both fixed point and Newton-Raphson schemes. In this research, the iteration was stopped when the norm of the difference between the generalized coordinates sets $\mathbf{q}_{j}$ and $\mathbf{q}_{j+1}$ obtained in two consecutive iterations of the solver was less than a certain threshold value. With this criterion the number of iterations in each integration time-step is not known a priori, which could compromise the ability of the method to deliver real-time performance. For this reason, the number of iterations of the solver was monitored during the simulations; it was found that it never went beyond ten. In practice, to make sure that the simulation complies with real-time execution requirements, a fixed number of iterations could be used as termination criterion.

\subsection{Implementation details}

The efficiency of a given multibody dynamics formulation critically depends on implementation aspects. For this study, the multibody formulations described in Section 3.2 were implemented using the techniques reported in [21]. Modelling reel-in operations as changes in cable length using the rheonomic constraints in Eqs. (5b) allows one to keep the sparsity pattern of the Jacobian matrix unaltered during the entire simulation. This, in turn makes the sparsity pattern of matrix $\mathbf{A}=\mathbf{M}+\mathbf{\Phi}_{\mathbf{q}}^{\mathrm{T}} \alpha \boldsymbol{\Phi}_{\mathbf{q}}$ constant as well. Matrix $\mathbf{A}$ is the leading matrix of the linear systems that need be solved to obtain the derivatives of the system variables $\mathbf{y}$, as shown in Eqs. (14a) and (16), and implied in Eq. (21). The linear solver can then reuse the original symbolic factorization of $\mathbf{A}$ at every time-step during the whole motion, while updating its numerical 
values via optimized implementations of the rank- $k$ update of the symmetric matrix $\boldsymbol{\Phi}_{\mathbf{q}}^{\mathrm{T}} \alpha \mathbf{\Phi}_{\mathbf{q}}$ and its addition to $\mathbf{M}$. Additionally, matrix $\mathbf{A}$ is guaranteed to be symmetric and positive-definite, provided that a suitable value of the penalty factor $\alpha$ has been chosen, which enables the use of solvers based on sparse Cholesky factorization like CHOLMOD [9], the one selected for this research.

\section{Test examples}

The accuracy, applicability conditions, and efficiency of the methods described in Sections 2 and 3 were tested in the forward-dynamics simulation of two test cases.

\subsection{Single cable}

The first test example is shown in Fig. 5. A cable of length $L$ and mass $m$ is towed at constant velocity $-v_{f}$. This cable is made up of a steel core and polymer fibres, as it is common in towed fishing gear applications. A point mass $m_{p}$ is attached to its free tip. This simple test isolates the effect of the retraction process on the time scale of the system dynamics and enables the performance analysis of both multibody and spring-based models, as well as the proposed numerical improvement strategies. In addition, it can be reproduced easily, thus allowing the comparison of results of different research works.

The physical parameters of the problem are summarized in Table 3. Since the spring and the multibody models treat the axial cable stiffness $E A$ in dissimilar ways, three different values of this parameter were used to assess its effect on the performance of the computational methods.

Initially, the cable is aligned with the global $x$-axis. The test consists of a 300-s forwarddynamics simulation of a reel-in manoeuvre, in which the cable is wound around the winch drum at a constant length-reduction rate of $v_{w}=100 \mathrm{~m} / \mathrm{min}$. During motion, the system is subject to the action of gravity, $g=9.81 \mathrm{~m} / \mathrm{s}^{2}$ acting along the negative $y$-axis, hydrodynamic forces, and contact with the seabed. The values of the seabed stiffness and friction coefficient were obtained from [42]. 


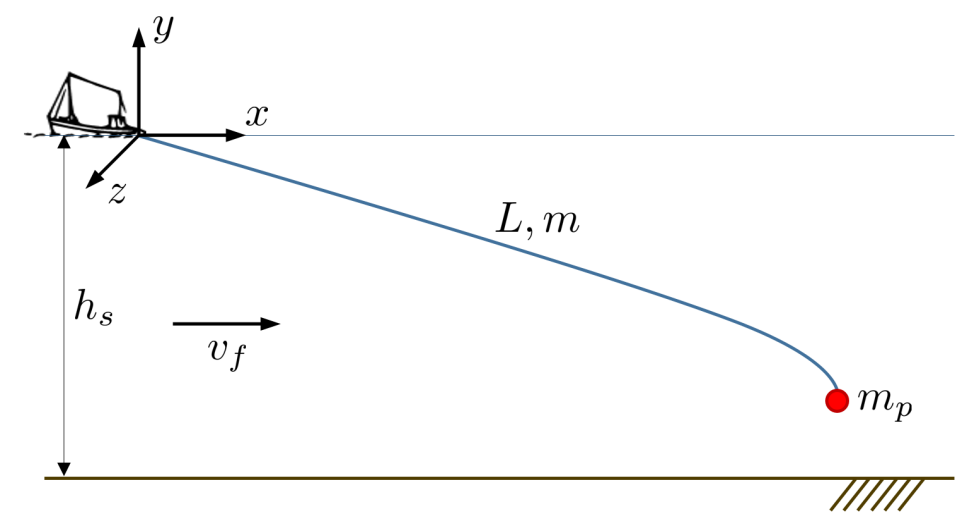

Figure 5: Single-cable example used as test problem

Table 3: Physical parameters of the single-cable example

\begin{tabular}{ll}
\hline Parameter & value \\
\hline Total cable mass, $m$ & $1000 \mathrm{~kg}$ \\
Total cable length, $L$ & $1000 \mathrm{~m}$ \\
Cable diameter, $D$ & $0.02 \mathrm{~m}$ \\
Cable stiffness, $E A$ & $10^{6}, 10^{7}, 10^{8} \mathrm{~N}$ \\
Towed mass, $m_{p}$ & $200 \mathrm{~kg}$ \\
Depth to seabed, $h_{s}$ & $-100 \mathrm{~m}$ \\
Seabed stiffness, $k_{s}$ & $10^{4} \mathrm{~N} / \mathrm{m}$ \\
Seabed friction coefficient, $\mu_{s}$ & 1 \\
Fluid density, $\rho$ & $1000 \mathrm{~kg} / \mathrm{m}^{3}$ \\
Normal drag coefficient, $C_{d}$ & 1.2 \\
Tangent friction coefficient, $C_{f}$ & 0.08 \\
Fluid velocity along $x, v_{f}$ & $1.5 \mathrm{~m} / \mathrm{s}$ \\
\hline
\end{tabular}

In order to represent the trawler motion while keeping the position of the winch fixed at the origin of the global reference frame, the fluid and the seabed were assigned a velocity $v_{f}$ in the opposite direction.

\subsection{Fishing trawl}

The second test example, shown in Fig. 6, consists in a simplified representation of a doublecable fishing trawl, with the configuration depicted in Fig. 1. Each of the two cables that link the net to the trawler has the same physical properties as the one described in Section 4.1. 


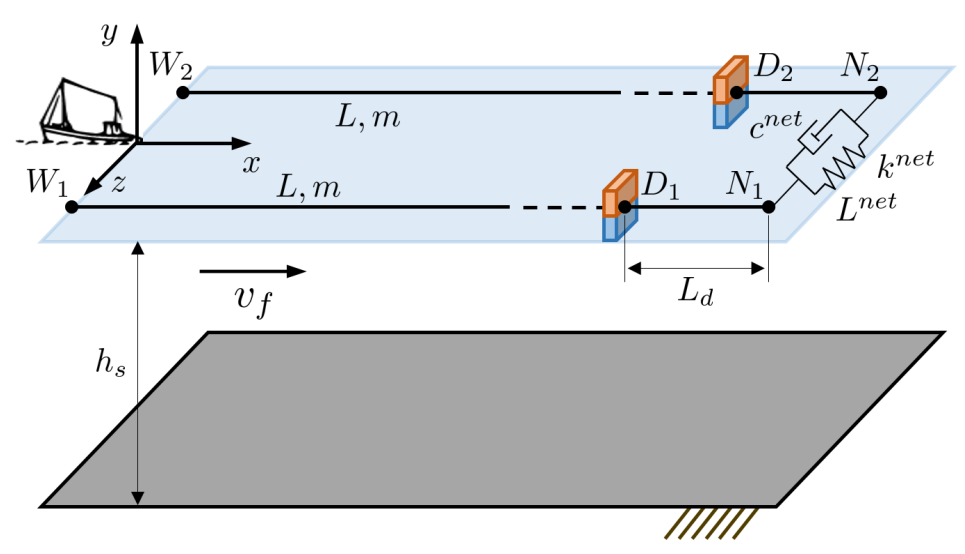

Figure 6: Schematic of the initial configuration of the fishing trawl

The fishing net is modelled as a mass-spring-damper system placed between the two opposite sides of the entrance to the net. This model is a simplification of a real fishing net, intended to make it possible to compare the performance of the different cable models. A detailed model of a fishing net would demand a high number of extra variables, thus hampering the efficiency comparison of the methods to simulate cables proposed in this research. The magnitude of the elastic and damping forces, $\tilde{f}_{e}^{n e t}$ and $\tilde{f}_{d p}^{n e t}$, introduced by this element are

$$
\begin{aligned}
& \widetilde{f}_{e}^{n e t}=E A^{n e t} \frac{s^{n e t}-L^{n e t}}{L^{n e t}}=k^{n e t}\left(s^{n e t}-L^{n e t}\right) \\
& \widetilde{f}_{d p}^{n e t}=-c^{n e t} v_{d p}^{n e t}
\end{aligned}
$$

where $s^{n e t}$ is the distance between the tips of the two cables $N_{1}$ and $N_{2}$, i. e., the horizontal spread, and $v_{d p}^{n e t}$ is the velocity of elongation of the element. The natural length of the element $L^{n e t}$ and its stiffness $E A^{\text {net }}$ were chosen to match the horizontal opening of a physical net. The damping coefficient $c^{\text {net }}$ was estimated by fitting the dynamic response of the mass-damperspring system with a detailed model of a fishing net [50] subjected to opposite horizontal loads on points $N_{1}$ and $N_{2}$.

The spring-damper element also experiences normal and tangent hydrodynamic forces, whose magnitudes are respectively

$$
\begin{aligned}
& \widetilde{f}_{d n}^{n e t}=\frac{1}{2} \rho C_{d}^{n e t} A^{n e t}\left(v_{n}^{n e t}\right)^{2} \\
& \widetilde{f}_{d t}^{n e t}=\frac{1}{2} \rho C_{f}^{n e t} A^{n e t}\left(v_{t}^{n e t}\right)^{2}
\end{aligned}
$$


where $v_{n}^{\text {net }}$ and $v_{t}^{\text {net }}$ are the magnitudes of the normal and tangential components of the velocity of the fluid relative to the spring-damper element and $C_{d}^{\text {net }}$ and $C_{f}^{\text {net }}$ are the normal and tangential drag coefficients. The effective hydrodynamic area $A^{\text {net }}$ is approximated as the area of a rectangle defined by the nominal horizontal and vertical net opening.

The doors are modelled as point masses placed on nodes $D_{1}$ and $D_{2}$, at a distance $L_{d}$ from the end nodes of the cables, $N_{1}$ and $N_{2}$, at which the net is connected to the cables. Particularized expressions of the hydrodynamic forces are used to evaluate the drag and lift forces undergone by these elements. These expressions assume that the hydrodynamic forces are independent of the orientation of the door $[46,50]$

$$
\begin{aligned}
\widetilde{f}_{d}^{\text {door }} & =\frac{1}{2} \rho C_{d}^{\text {door }} A^{\text {door }}\left(v^{\text {door }}\right)^{2} \\
\widetilde{f}_{l}^{\text {door }} & =\frac{1}{2} \rho C_{l}^{\text {door }} A^{\text {door }}\left(v^{\text {door }}\right)^{2}
\end{aligned}
$$

where $C_{d}^{\text {door }}$ and $C_{l}^{\text {door }}$ are the drag and lift coefficients of the door, $A^{\text {door }}=h^{\text {door }} w^{\text {door }}$ is the door surface area, with $h^{\text {door }}$ and $w^{\text {door }}$ the height and width of the door respectively, and $v^{d o o r}$ is the magnitude of the fluid velocity relative to the door. The contact between the seabed and the doors is modelled with Eq. (12) for the tangent direction, replacing Eq. (11) with

$$
\tilde{f}_{c n}^{d o o r}=-k_{s}\left(y_{i}-h_{s}-h^{d o o r} / 2\right)
$$

which takes into account the door height $h^{\text {door }}$ in the computation of the indentation.

The initial configuration of the system is sketched in Fig. 6. At $t=0$ the two cables are fully deployed and aligned in parallel with the global $x$-axis at coordinates $z=5 \mathrm{~m}$ and $z=-5 \mathrm{~m}$. Points $W_{1}$ and $W_{2}$ denote the location of the two winches, which are mounted on the trawler. The introduction of doors in the assembly makes the system motion 3D. Hydrodynamics lift forces on the doors ensure the correct deployment of the fishing net, pulling points $N_{1}$ and $N_{2}$ apart from each other.

The physical parameters of the simulation are the ones shown in Table 3, plus additional ones for the elements incorporated in the trawl, i.e., net and doors, contained in Table 4. The equivalent parameters of the net are based on a bottom trawl model provided by a Spanish company [13]. Similarly, the door parameters correspond to model Ovalfoil OF-13 by door maker Morgère S. A. S. [37]. 
Table 4: Physical parameters of the double-cable assembly

\begin{tabular}{ll}
\hline Parameter & value \\
\hline Mass of the net, $m^{\text {net }}$ & $3000 \mathrm{~kg}$ \\
Equivalent stiffness of the net, $k^{\text {net }}$ & $10^{3} \mathrm{~N} / \mathrm{m}$ \\
Equivalent natural length of the net, $L^{\text {net }}$ & $10 \mathrm{~m}$ \\
Equivalent damping of the net, $c^{\text {net }}$ & $10^{4} \mathrm{Ns} / \mathrm{m}$ \\
Equivalent area of the net, $A^{\text {net }}$ & $20 \mathrm{~m}^{2}$ \\
Normal drag coefficient of the net, $C_{d}^{\text {net }}$ & 1.2 \\
Tangent friction coefficient of the net, $C_{f}^{\text {net }}$ & 0.08 \\
Mass of the door, $m^{\text {door }}$ & $2100 \mathrm{~kg}$ \\
Door height, $h^{\text {door }}$ & $2.69 \mathrm{~m}$ \\
Door width, $w^{\text {door }}$ & $3.45 \mathrm{~m}$ \\
Drag coefficient of the door, $C_{d}^{\text {door }}$ & 0.76 \\
Lift coefficient of the door, $C_{l}^{\text {door }}$ & 1.76 \\
Initial $x$-distance between door and net, $L_{d}$ & $10 \mathrm{~m}$
\end{tabular}

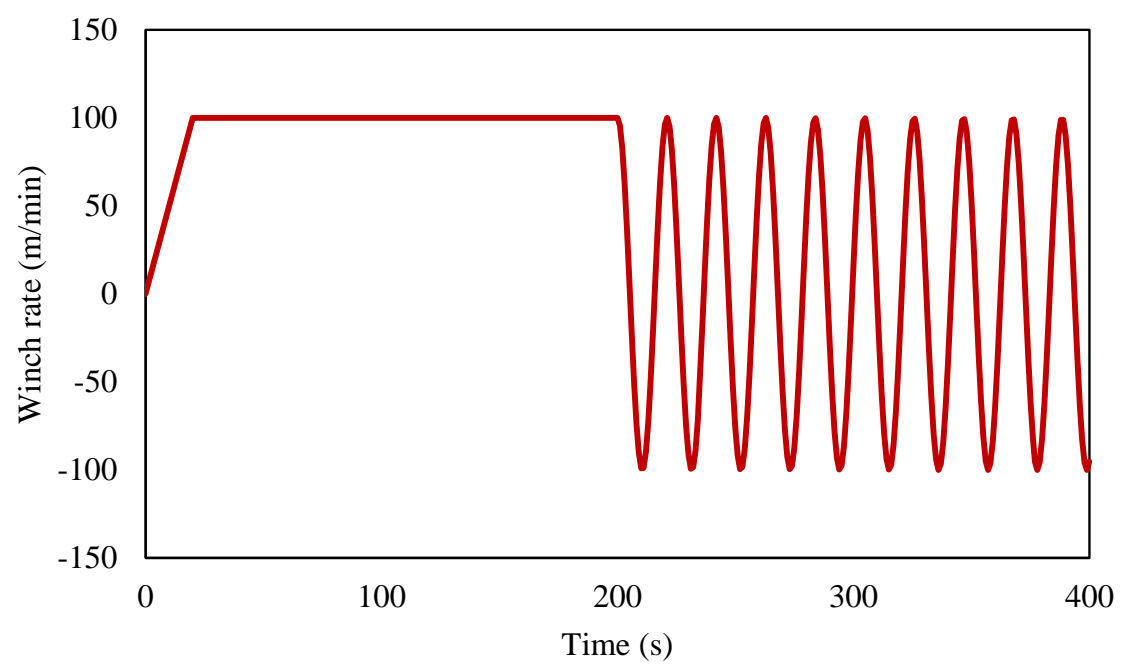

Figure 7: Winch rate in Manoeuvre II, $\omega_{w}=0.3 \mathrm{rad} / \mathrm{s}$

Two manoeuvres were simulated with this assembly:

1. Manoeuvre I: A 300-s reel-in operation in which the trawler speed $v_{f}$ and the winch velocity $v_{w}$ were increased linearly from zero up to their steady-state values, $v_{f}=1.5 \mathrm{~m} / \mathrm{s}$ and $v_{w}=100 \mathrm{~m} / \mathrm{min}$, during the first $20 \mathrm{~s}$ of the motion. This was done to avoid unrealistic overshoots in the cable tension at the motion onset.

2. Manoeuvre II: A 400-s motion identical to the previous one until $t=200 \mathrm{~s}$. From this point 
on, the winch velocity was commanded to follow a sinusoidal profile with amplitude $v_{w}$ and frequency $\omega_{w}$, as shown in Fig. 7. Such a profile imitates the actions of a winch control system during the towing stage of the fishing process.

\section{Results and discussion}

The test problems described in Section 4 were simulated modelling the cable both as a chain of linear springs and as a multibody system. All simulations were carried out in an Intel i7-4790K processor at $4.0 \mathrm{GHz}$ with $8 \mathrm{~GB}$ of RAM, running Windows 10 Pro. The solution obtained with the unimproved, original linear-spring model, validated with experiments in [50], was taken as reference in the numerical experiments shown next. The solutions delivered by the other methods were considered correct when the trajectories of the points and the cable tension at the winch matched those obtained with this reference method. In order to quantify the deviation of these magnitudes with respect to the reference, a relative error for a magnitude $y$ in each simulation was defined as [20]

$$
e_{\text {total }}=\sqrt{\frac{1}{n_{s}} \sum_{i=1}^{n_{s}}\left(\frac{\left|y\left(t_{i}\right)-y^{r e f}\left(t_{i}\right)\right|}{y^{r e f}\left(t_{i}\right)}\right)^{2}}
$$

where $t_{i}$ is the timestamp of each magnitude sampling, $n_{s}$ is the total number of sampling points, and $y^{r e f}$ is the reference solution. Simulations were considered correct if the deviations remained below a $10 \%$ error for the entire simulation.

\subsection{Single cable}

The motion of the first example was simulated discretizing the cable with $N=100$ elements, using the linear spring model with $E A=10^{6} \mathrm{~N}$ and the multibody methods in Table 2. None of the numerical improvements described in Section 2.4 were used in these initial simulations. The simulation outputs were the $x$ - and $y$-coordinates of the cable tip, at which the point mass $m_{p}$ was attached, and the cable tension.

Fig. 8 shows that both simulation strategies, linear spring and multibody, yielded a very simi- 

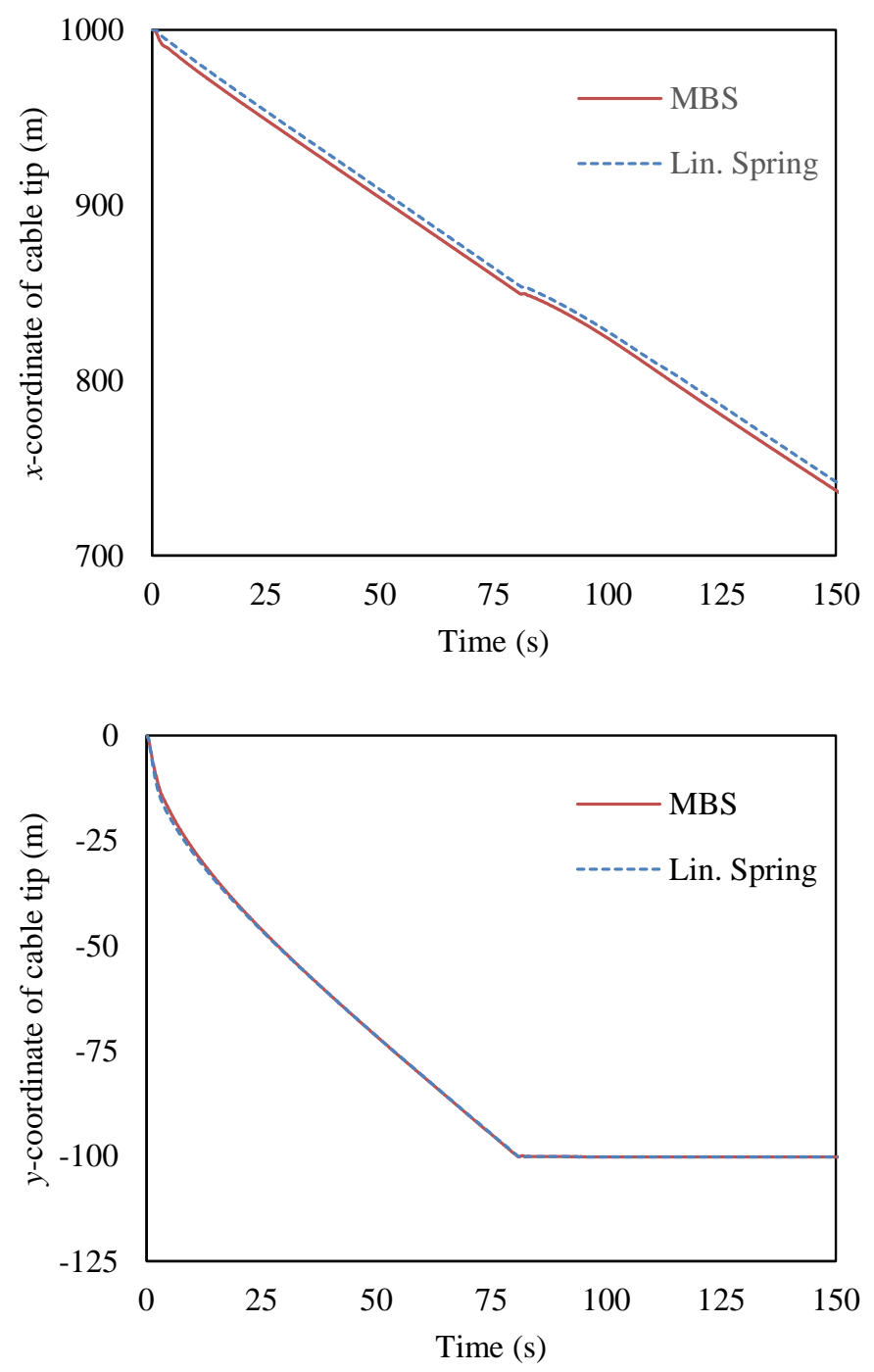

Figure 8: $x$ - and $y$-coordinates of point mass $m_{p}$ during the simulation of the reel-in manoeuvre with the single cable

lar motion of the cable tip. As mentioned in Section 2.2, modelling the cable with the multibody approach is equivalent to assuming that the cable has infinite axial stiffness. This removes the elongation of the cable due to axial strain and introduces a slight difference with respect to the position of the tip calculated with the spring model. This difference remained below $0.6 \%$ of the initial length of the cable for this manoeuvre.

As shown in Fig. 9, the simulation with the multibody approach correctly evaluated the cable tension as well. It is worth mentioning that the infinite axial stiffness of the cable introduces an overshoot during the initial seconds of the motion. Moreover, the output became noisy and had to be smoothed by means of a moving average; the output cable tension $\xi_{k}^{*}$ at time-step 


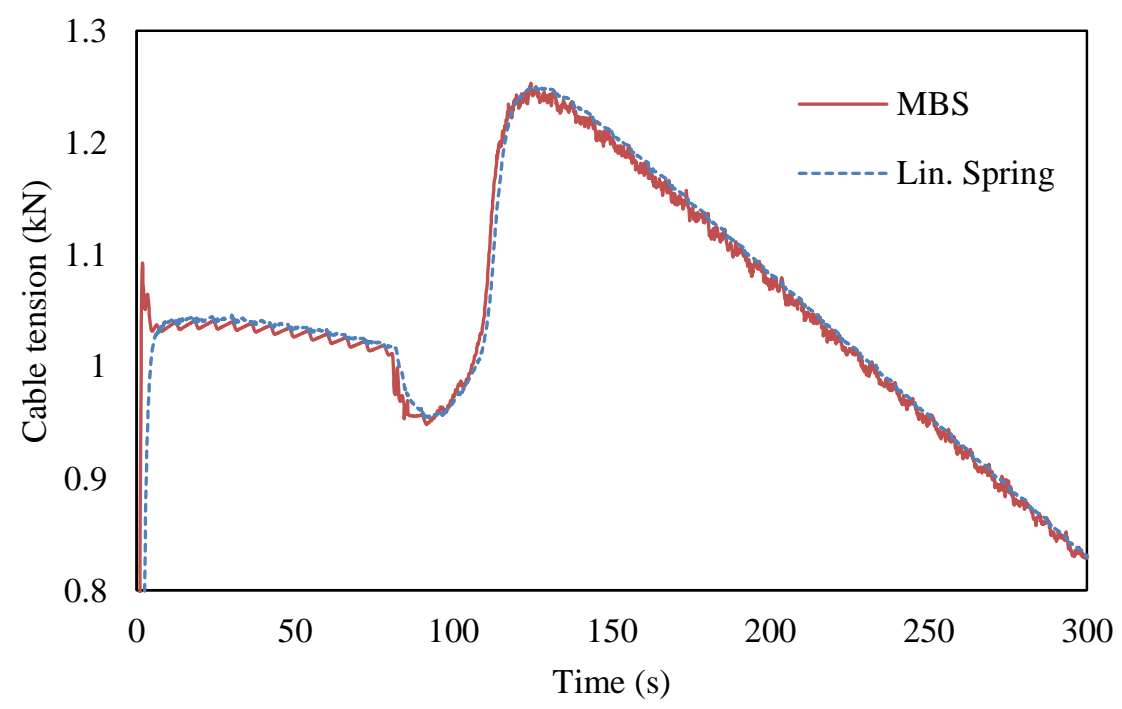

Figure 9: Cable tension during the simulation of the reel-in manoeuvre with the single cable

$k$ was evaluated as the average of the last $n_{p}$ values of cable tension yielded by the solver, $\xi_{k}, \ldots, \xi_{k-n_{p}+1}$

$$
\xi_{k}^{*}=\frac{1}{n_{p}} \sum_{i=0}^{n_{p}-1} \xi_{k-i}
$$

The simulation was repeated using the numerical improvement strategies described in Section 2.4 and no significant differences in the motion or cable tension were observed. The selection of a particular multibody algorithm among those in Table 2 did not alter the output of the simulation either.

The use of numerical improvements for the linear spring model introduced in Section 2.4 enabled considerable reductions of simulation runtime. Methods No. 1, No. 2, and No. 3 were 7, 26, and 117 times faster than the unimproved approach, respectively. The three strategies curtailed the increase of the natural frequency of the cable elements during the reel-in process, allowing larger step-sizes than the uncorrected method, e.g., from $0.016 \mathrm{~ms}$ to $2 \mathrm{~ms}$ in strategy No. 3. These improvements in computational efficiency were proportional to the step-size increment since convergence was achieved with at most 2 iterations in each step of the fixed point scheme.

The simulation was later repeated with higher values of cable stiffness, $E A=10^{7} \mathrm{~N}$ and 


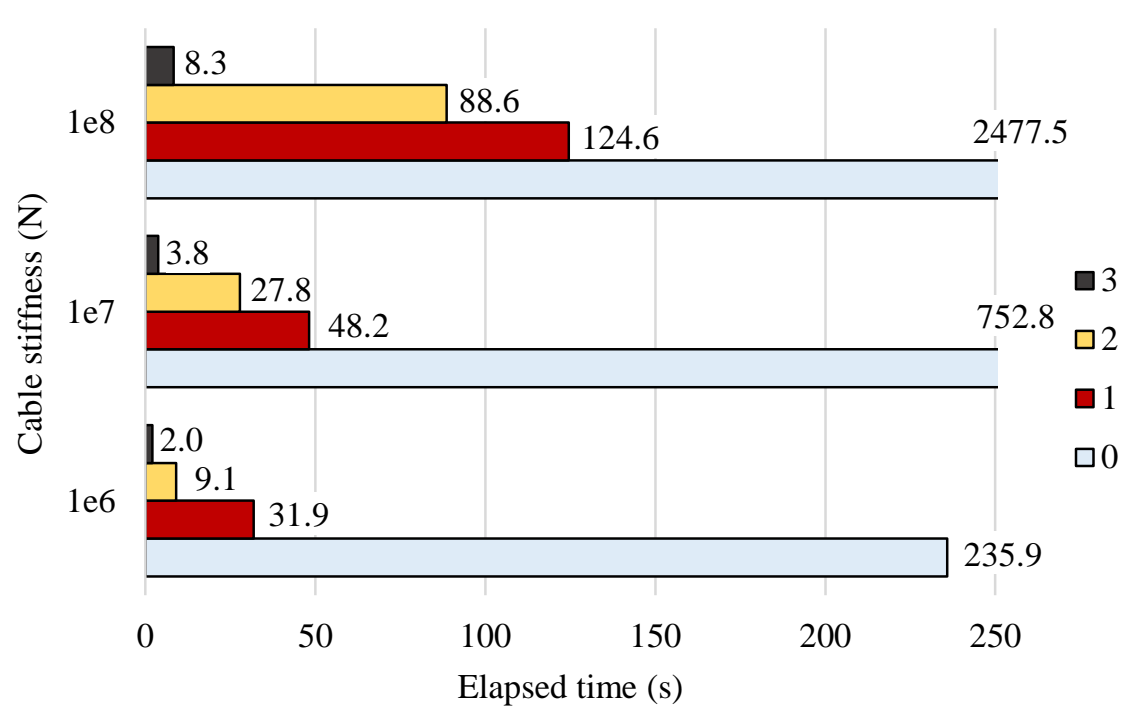

Figure 10: Effect of the numerical improvement methods in Table 1 on the elapsed time in the simulation of the single-cable motion with the linear spring model, $N=100$

$E A=10^{8}$ N. As shown in Fig. 10, although the execution time augmented with cable stiffness, the advantage of the methods over the unimproved one also increased, reaching 20 times faster for strategy No.1, about 27 times for No. 2, and 300 times faster for method No.3. Raising the cable stiffness brought the trajectory of point mass $m_{p}$ closer to the one obtained with multibody methods. Cable tension remained practically unaltered.

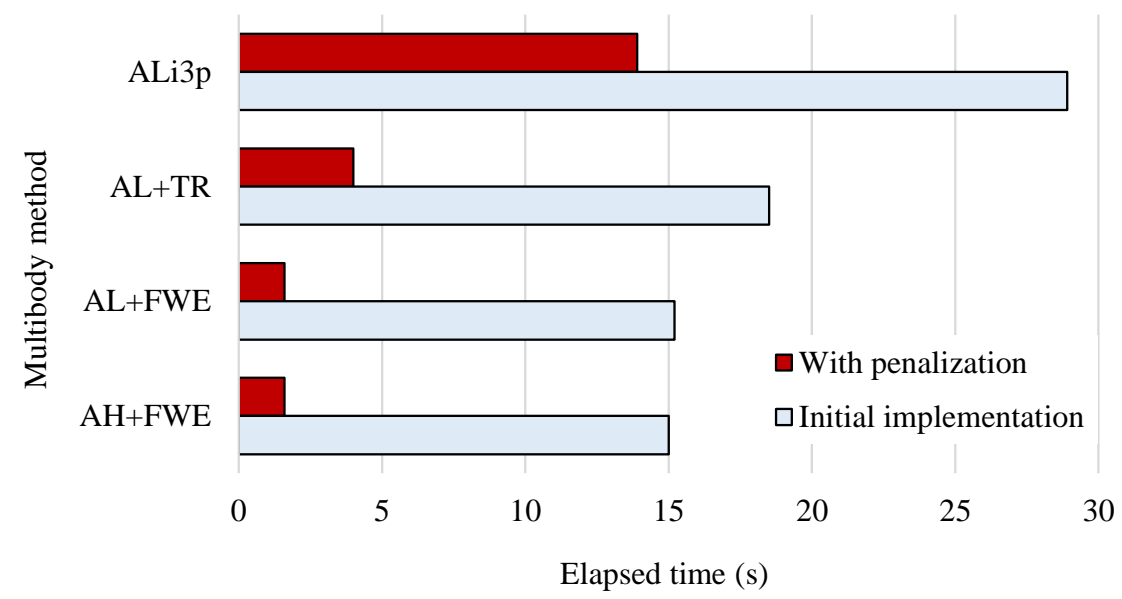

Figure 11: Effect of the acceleration-level dynamics penalization on the elapsed time in the simulation of the single-cable motion with the multibody model, $N=100$

Regarding the multibody model of the cable, all the evaluated formalisms delivered execution times under $30 \mathrm{~s}$. The acceleration-level penalization in Eq. (8) managed to improve the 
efficiency of the methods, being up to 6 times faster in the case of approaches with single-step integration routines, as shown in Fig. 11. The maximum value of the penalty factor $p_{\alpha}$ ranged between $10 m_{i}$ and $25 m_{i}$, depending on the formulation.

Additional tests were run to determine the ability of the proposed methods to carry out real-time simulations when the cable is discretized using more elements. Besides increasing the problem size, raising $N$ diminishes the length and inertia of the cable segments. For $N=1000$, i.e., $L_{i}=1 \mathrm{~m}$ and $m_{i}=1 \mathrm{~kg}$, the moment of inertia of a cable segment is $0.083 \mathrm{kgm}^{2}$, three orders of magnitude smaller than for $N=100$. This results in faster system dynamics and, as a consequence, it is necessary to reduce the integration step-size to prevent the simulation from turning unstable.

Table 5: Integration step-sizes and elapsed times in the simulation of the single-cable example with the linear spring model and different numerical improvements

\begin{tabular}{|c|c|c|c|c|c|c|}
\hline & $N$ & 100 & 250 & 500 & 750 & 1000 \\
\hline \multicolumn{7}{|c|}{$E A=10^{6} \mathrm{~N}$} \\
\hline & $\Delta t(\mathrm{~ms})$ & 0.12 & 0.12 & 0.10 & 0.09 & 0.08 \\
\hline \multirow[t]{2}{*}{ Meth. 1} & elap. (s) & 32.0 & 78.0 & 180.0 & 318.2 & 457.4 \\
\hline & $\Delta t(\mathrm{~ms})$ & 0.5 & 0.3 & 0.2 & 0.15 & 0.15 \\
\hline \multirow[t]{2}{*}{ Meth. 2} & elap. (s) & 9.1 & 33.1 & 94.7 & 192.7 & 278.8 \\
\hline & $\Delta t(\mathrm{~ms})$ & 2 & 1.2 & 0.8 & 0.6 & 0.5 \\
\hline Meth. 3 & elap. (s) & 2.0 & 7.4 & 22.1 & 45.9 & 71.8 \\
\hline \multicolumn{7}{|c|}{$E A=10^{7} \mathrm{~N}$} \\
\hline & $\Delta t(\mathrm{~ms})$ & 0.08 & 0.06 & 0.042 & 0.035 & 0.03 \\
\hline \multirow[t]{2}{*}{ Meth. 1} & elap. (s) & 48.2 & 172.6 & 428.3 & 836.0 & 1202.9 \\
\hline & $\Delta t(\mathrm{~ms})$ & 0.15 & 0.09 & 0.06 & 0.05 & 0.045 \\
\hline \multirow[t]{2}{*}{ Meth. 2} & elap. (s) & 27.8 & 106.5 & 310.0 & 589.0 & 879.1 \\
\hline & $\Delta t(\mathrm{~ms})$ & 1 & 0.6 & 0.32 & 0.22 & 0.17 \\
\hline Meth. 3 & elap. (s) & 3.8 & 14.5 & 54.6 & 125.0 & 209.2 \\
\hline \multicolumn{7}{|c|}{$E A=10^{8} \mathrm{~N}$} \\
\hline & $\Delta t($ & 0.03 & 0.02 & 0.012 & 0.009 & 0.007 \\
\hline \multirow[t]{2}{*}{ Meth. 1} & elap. (s) & 124.6 & 575.5 & 1479.8 & 3068.2 & 5114.3 \\
\hline & $\Delta t(\mathrm{~ms})$ & 0.045 & 0.03 & 0.02 & 0.015 & 0.012 \\
\hline \multirow[t]{2}{*}{ Meth. 2} & elap. (s) & 88.6 & 330.8 & 949.5 & 1943.4 & 3165.2 \\
\hline & $\Delta t(\mathrm{~ms})$ & 0.45 & 0.22 & 0.11 & 0.07 & 0.055 \\
\hline Meth. 3 & elap. (s) & 8.3 & 39.7 & 158.4 & 394.2 & 649.0 \\
\hline
\end{tabular}

Table 5 shows the integration step-sizes, $\Delta t$, and elapsed times invested in the simulation of 
the example with the linear spring model and the numerical improvement methods described in Section 2.4. As expected, as the number of elements $N$ used in the discretization grows smaller step-sizes are necessary to complete the simulation successfully. Stiffer cables required smaller step-sizes as well and gave rise to increases in the computation times. The third method, which combines spring softening with fixing the mass of the cable elements during reel-in, showed the best performance, making it possible to attain real-time performance in most cases.

Table 6: Integration step-sizes and elapsed times in the simulation of the single-cable example with the multibody methods and correction of the dynamics of the retracted segments

\begin{tabular}{lcccccc}
\hline & $N$ & 100 & 250 & 500 & 750 & 1000 \\
\hline ALi3p & $\Delta t$ (ms) & 2 & 2 & 2 & 1 & 0.7 \\
& elap. (s) & 13.9 & 34.1 & 67.9 & 204.3 & 273.8 \\
\hline \multirow{2}{*}{ AL+TR } & $\Delta t$ (ms) & 10 & 6 & 4 & 3 & 1 \\
& elap. (s) & 4.0 & 14.0 & 41.2 & 80.7 & 150.6 \\
\hline \multirow{2}{*}{ AL+FWE } & $\Delta t$ (ms) & 10 & 8 & 5 & 4 & 3 \\
& elap. (s) & 1.6 & 4.8 & 14.9 & 27.6 & 49.3 \\
\hline AH+FWE & $\Delta t$ (ms) & 10 & 8 & 5 & 3 & 2 \\
& elap. (s) & 1.6 & 4.8 & 15.1 & 36.5 & 73.5 \\
\hline
\end{tabular}

The elapsed times obtained with multibody algorithms, together with the required integration step-sizes, are detailed in Table 6. Acceleration-level penalization of the dynamics of retracted cable segments was used in all cases. Although real-time performance was achieved in all cases, formalisms with single-step, explicit integrators were significantly more efficient, especially for discretizations with $N>500$ elements.

Figure 12 compares the performance of the linear spring and multibody methods in the simulation of the single cable example. Only the most efficient algorithms are depicted in the figure, namely the AH+FWE and AL+FWE multibody methods and integration with numerical improvement No. 3 for the linear spring model. Moreover, this figure highlights the fact that the elapsed time does not increase linearly with $N$, as a consequence of the need for a shorter step-size $\Delta t$ when the cable segments become smaller. 


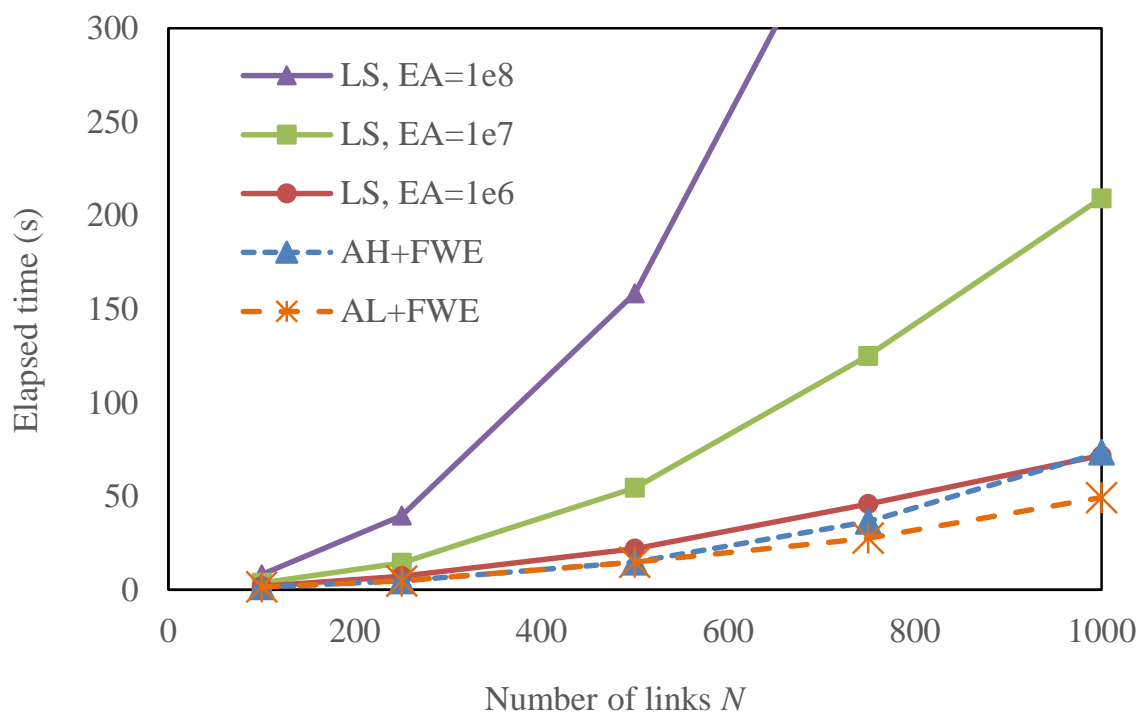

Figure 12: Elapsed times in the simulation of the single cable motion with the two most efficient multibody formalisms and the linear spring model with numerical improvement No. 3 and three different values of cable stiffness

\subsection{Fishing trawl, Manoeuvre I}

Manoeuvre I was simulated with the spring and multibody models using the numerically improved algorithms described in Section 2.4.

Fig. 13 shows that both methods correctly captured the effect of introducing doors in the assembly. As the reel-in operation progressed, the horizontal spread increased due to the hydrodynamic forces acting at the doors. The change of trajectory that took place around $x=850 \mathrm{~m}$ was caused by the sudden introduction of contact forces when the doors reached the seabed. The effect of changing the axial stiffness of the cable on the trajectory was negligible.

The tension in cables, represented in Fig. 14, reached its maximum value at $t \approx 25 \mathrm{~s}$, once the winch rate $v_{w}$ and the trawler velocity $v_{f}$ reached their maximum, steady-state values. From this moment on, the tension steadily decreased due to the reduction in cable length and, therefore, cable weight and resultant hydrodynamic forces. At $t \approx 100 \mathrm{~s}$ the doors reached the seabed and friction forces introduced a significant increment, about $11 \%$, in cable tension. The numerical improvement approaches for the linear spring model did not introduce significant modifications in the tension plot.

It was confirmed that the axial flexibility of the cable imposes a limit on the applicability 

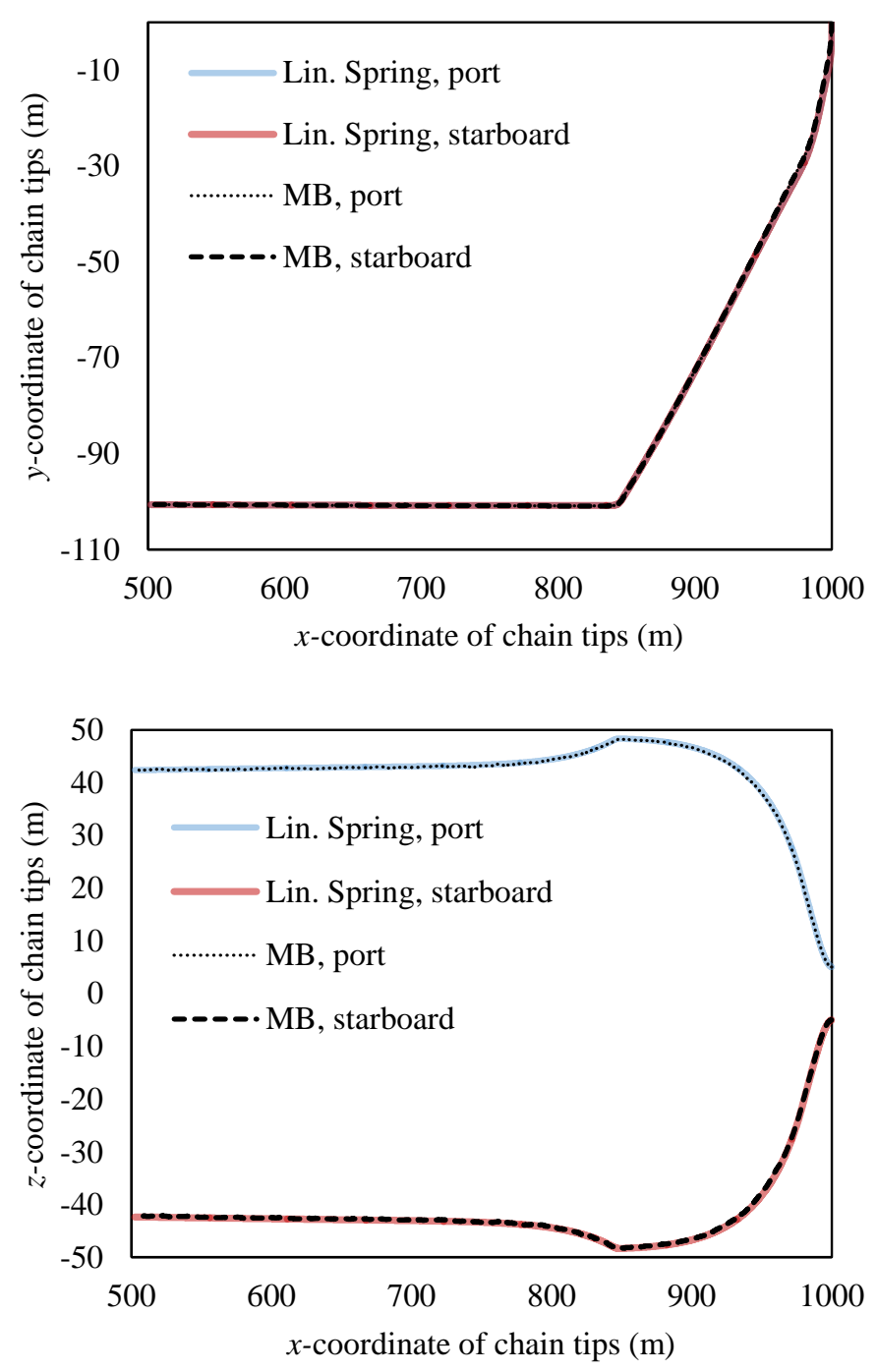

Figure 13: Projection on the $x-y$ plane and on the $x-z$ plane of the trajectory followed by the tips of the double cable during Manoeuvre I, simulated with linear-spring and multibody models

of multibody modelling, as highlighted by Fig. 14. In this figure, three different values of the axial stiffness of the cable are represented, corresponding to $E A=10^{9} \mathrm{~N}, E A=10^{8} \mathrm{~N}$, and $E A=10^{7} \mathrm{~N}$. The plots show that multibody modelling reproduced accurately these curves when the axial stiffness of the cable was high enough, $E A \geq 10^{8} \mathrm{~N}$. However, lower values of $E A$ resulted in larger axial strains, which in turn modified the cable dynamics and brought them further away from the ideally rigid behaviour predicted by the multibody simulation. Depending on the application the prediction could still be considered acceptable for the case in which $E A=10^{7} \mathrm{~N}$, especially after the initial transient has taken place, once the contact between the cables and the seabed occurs at $t \approx 100 \mathrm{~s}$. However, the results can no longer be considered correct if $E A<10^{7} \mathrm{~N}$. 


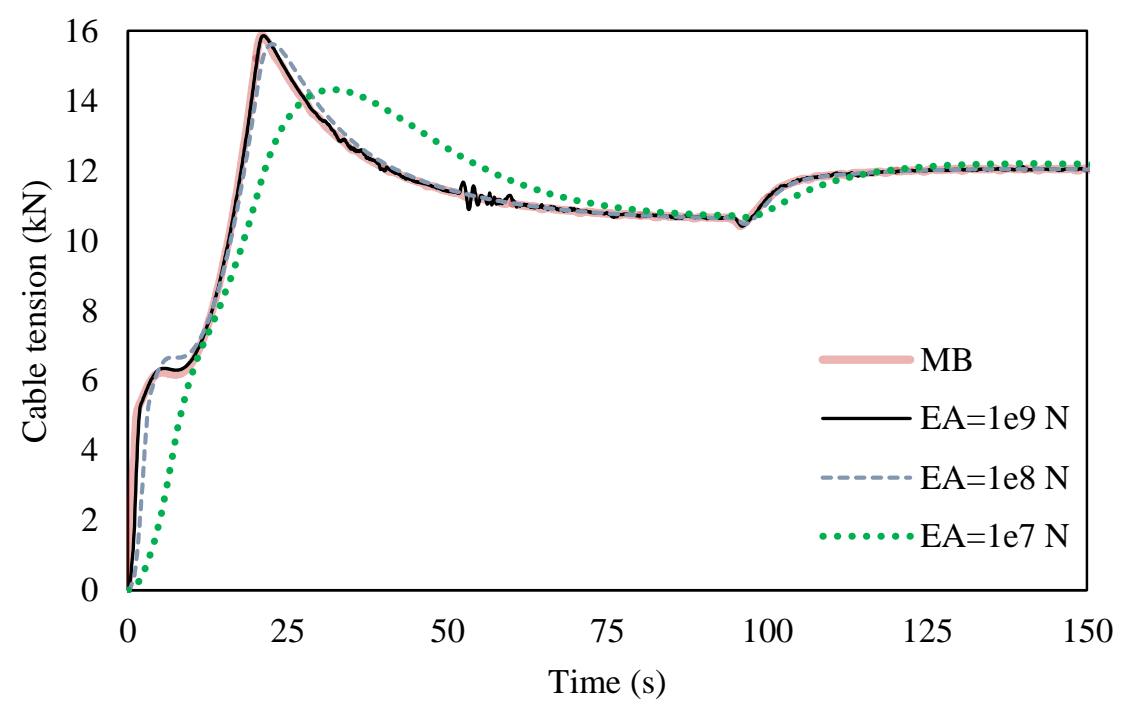

Figure 14: Cable tension during Manoeuvre I obtained with the multibody model (MB) and the linear spring approach with different values of the cable axial stiffness

Note that the multibody model predicted the correct tension also with low $E A$ values in the single-cable example, because in that case the cable tension was about 15 times smaller than in the fishing trawl. The obtained results suggest that the use of the multibody method should be restrained to problems in which cable tension does not exceed $E A \cdot 10^{-3}$.

Tables 7 and 8 show the elapsed times during the simulation of the manoeuvre with the linear spring and the multibody models. The loads that the cable undergoes in this double-cable assembly are one order of magnitude larger than in the previous test; moreover, the problem now involves $6 N$ variables and $2 N$ constraints when each cable is modelled with $N$ rigid segments.

Numerical improvement No. 3 delivered the best performance with the linear spring model, followed by method No. 2. Simulating cables with higher axial stiffness $E A$ made it necessary to reduce the integration step-size; the simulation of the cable with $E A=10^{8} \mathrm{~N}$ triplicated the execution time of that with $E A=10^{7} \mathrm{~N}$. As it happened with the previous example, increasing the number of cable segments caused a superlinear increment of the elapsed time, as the integration step-size had to be decreased. In spite of this, real-time simulation of the manoeuvre can be achieved with both the multibody and the linear spring models. Among multibody algorithms, those with single-step explicit integrator delivered again the fastest performances. 
Table 7: Integration step-sizes and elapsed times in the simulation of Manoeuvre I with the fishing trawl example and the linear spring model

\begin{tabular}{|c|c|c|c|c|c|}
\hline & $N$ & 100 & 200 & 250 & 500 \\
\hline \multicolumn{6}{|c|}{$E A=10^{7} \mathrm{~N}$} \\
\hline & $\Delta t(\mathrm{~ms})$ & 0.09 & 0.07 & 0.06 & 0.045 \\
\hline \multirow[t]{2}{*}{ Method 1} & elapsed (s) & 108.1 & 270.5 & 392.6 & 1132.0 \\
\hline & $\Delta t(\mathrm{~ms})$ & 0.15 & 0.09 & 0.09 & 0.07 \\
\hline \multirow[t]{2}{*}{ Method 2} & elapsed (s) & 59.4 & 185.6 & 239.5 & 689.0 \\
\hline & $\Delta t(\mathrm{~ms})$ & 1 & 0.8 & 0.65 & 0.3 \\
\hline Method 3 & elapsed (s) & 8.0 & 19.9 & 34.8 & 126.7 \\
\hline \multicolumn{6}{|c|}{$E A=10^{8} \mathrm{~N}$} \\
\hline & $\Delta t(\mathrm{~ms})$ & 0.032 & 0.023 & 0.019 & 0.015 \\
\hline \multirow[t]{2}{*}{ Method 1} & elapsed (s) & 312.1 & 895.4 & 1041.7 & 3756.4 \\
\hline & $\Delta t(\mathrm{~ms})$ & 0.045 & 0.035 & 0.03 & 0.02 \\
\hline \multirow[t]{2}{*}{ Method 2} & elapsed (s) & 192.5 & 550.1 & 745.1 & 2089.4 \\
\hline & $\Delta t(\mathrm{~ms})$ & 0.5 & 0.25 & 0.2 & 0.1 \\
\hline Method 3 & elapsed (s) & 15.9 & 62.9 & 98.4 & 378.5 \\
\hline
\end{tabular}

Table 8: Integration step-sizes and elapsed times in the simulation of Manoeuvre I with the fishing trawl example and the multibody methods

\begin{tabular}{|c|c|c|c|c|c|}
\hline & $N$ & 100 & 200 & 250 & 500 \\
\hline \multirow[t]{2}{*}{ ALi3p } & $\Delta t(\mathrm{~ms})$ & 3 & 2 & 2 & 2 \\
\hline & elapsed (s) & 18.9 & 55.4 & 69.1 & 158.6 \\
\hline \multirow[t]{2}{*}{$\mathrm{AL}+\mathrm{TR}$} & $\Delta t(\mathrm{~ms})$ & 5 & 5 & 4 & 1 \\
\hline & elapsed (s) & 6.6 & 27.2 & 42.5 & 155.8 \\
\hline \multirow[t]{2}{*}{$\mathrm{AL}+\mathrm{FWE}$} & $\Delta t(\mathrm{~ms})$ & 10 & 5 & 4 & 2 \\
\hline & elapsed (s) & 3.2 & 12.4 & 18.9 & 74.6 \\
\hline \multirow[t]{2}{*}{$\mathrm{AH}+\mathrm{FWE}$} & $\Delta t(\mathrm{~ms})$ & 9 & 4 & 3 & 1.5 \\
\hline & elapsed (s) & 3.6 & 15.2 & 25.1 & 99.6 \\
\hline
\end{tabular}

\subsection{Fishing trawl, Manoeuvre II}

Dynamic effects during Manoeuvre I had a negligible impact on the system motion after the completion of the initial transient phase. The aim of simulating Manoeuvre II is to study the 
ability of the different simulation methods to correctly represent the system response when the winch velocity $v_{w}$ changes rapidly and dynamic effects are no longer irrelevant. Such is the case of operations in which control algorithms are used to regulate cable tension. After $t=200 \mathrm{~s}$ in Manoeuvre II, the winch velocity follows a sinusoidal profile, alternating between reeling-in and letting-out stages at a given frequency $\omega_{w}$. In practical fishing applications, this frequency usually remains below $0.5 \mathrm{rad} / \mathrm{s}$. In this study, two values were tested, $\omega_{w}=0.1 \mathrm{rad} / \mathrm{s}$ and $\omega_{w}=0.3 \mathrm{rad} / \mathrm{s}$. These were selected to match winch controller data from real manoeuvres. In all these cases, it is expected that the axial stiffness of the cable affect the shape and values of the time history of the cable tension. The higher the cable stiffness, faster system response and higher tension peaks are to be expected. These effects will be more severe in systems in which the cable tension varies faster.

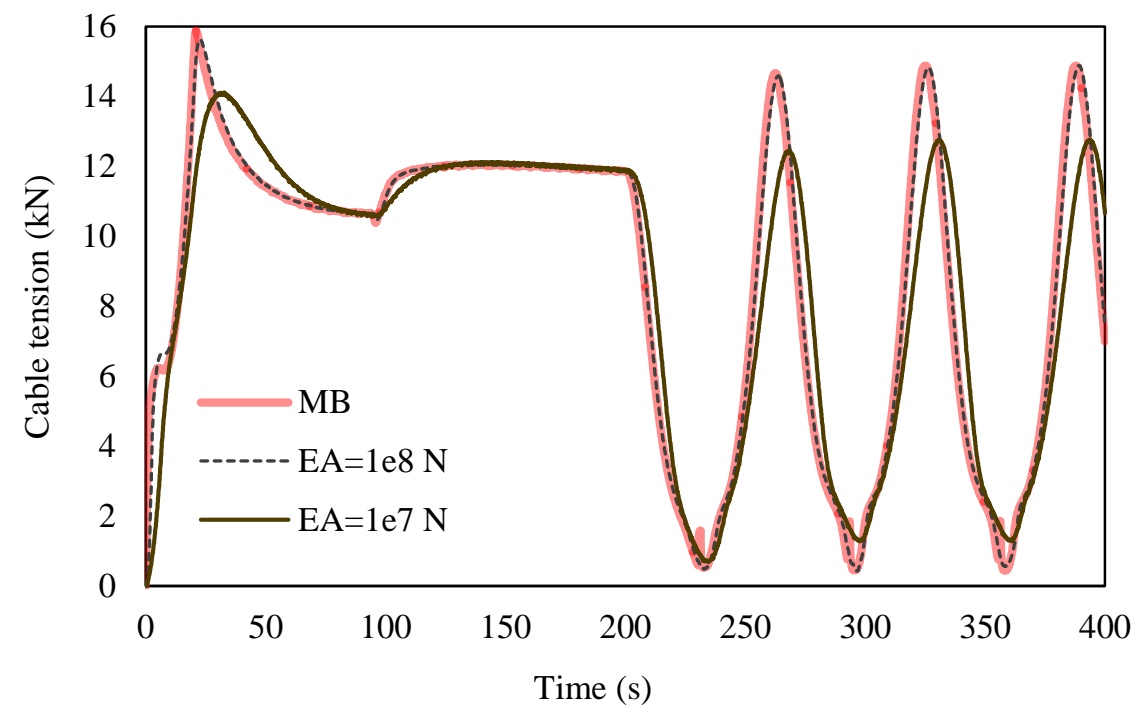

Figure 15: Cable tension during Manoeuvre II $\left(\omega_{w}=0.1 \mathrm{rad} / \mathrm{s}\right)$, obtained with the multibody and the linear spring approaches

Figures 15 and 16 show the tension values obtained during the simulation of the manoeuvre; the effect of the sinusoidal variation of $v_{w}$ is clearly noticeable after $t=200 \mathrm{~s}$. Fig. 15 shows the cable tension for $\omega_{w}=0.1 \mathrm{rad} / \mathrm{s}$. The multibody methods approximated with a high level of accuracy the tension undergone by stiff cables, with $E A=10^{8} \mathrm{~N}$. However, differences with respect to the linear spring model results were observed with softer cables, with $E A=10^{7}$ $\mathrm{N}$. The multibody methods predicted a faster response and $16 \%$ higher tension peaks. This is a consequence of neglecting the axial flexibility of the cable. The differences between both models were much more remarkable for $\omega_{w}=0.3 \mathrm{rad} / \mathrm{s}$, as shown in Fig. 16. In this case, the multibody 


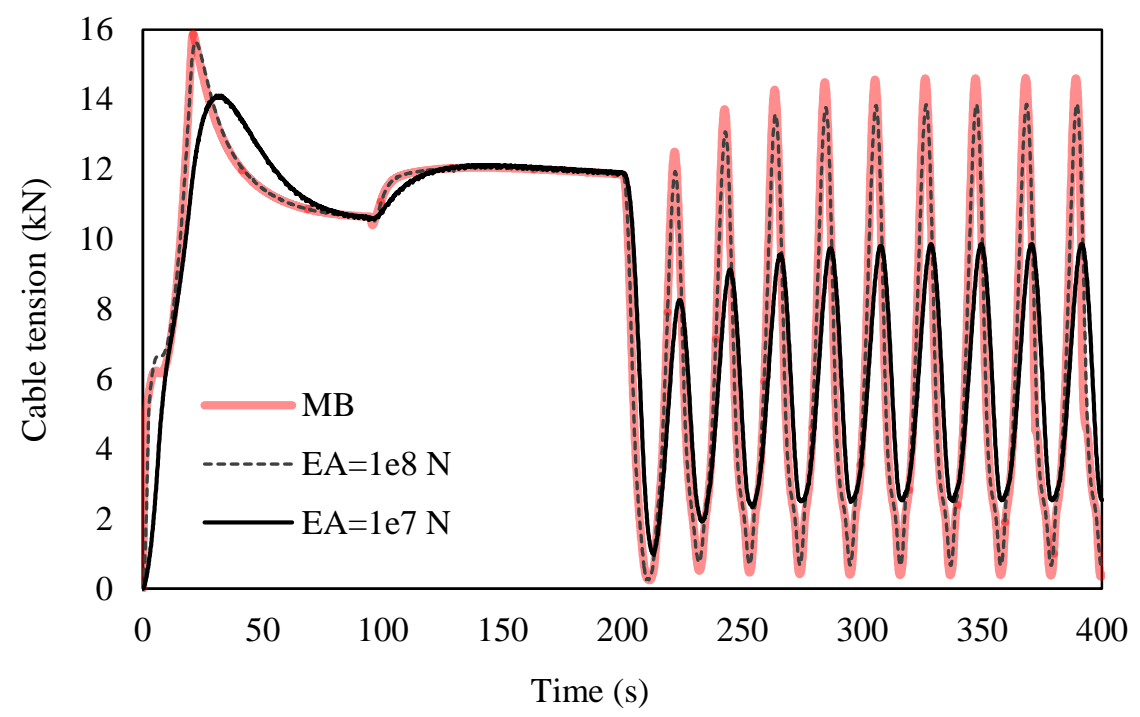

Figure 16: Cable tension during Manoeuvre II $\left(\omega_{w}=0.3 \mathrm{rad} / \mathrm{s}\right)$, obtained with the multibody and the linear spring approaches

models predicted tension peaks $8 \%$ higher than the linear spring with $E A=10^{8} \mathrm{~N}$. In the case of the cable with $E A=10^{7} \mathrm{~N}$, however, the amplitude of the oscillations calculated with the multibody methods was twice the value obtained with the linear spring model.

The above results confirmed that it is important to consider the axial stiffness of the cable to obtain accurate values of the tension during fast-dynamics manoeuvres. Among the numerical improvements described in Section 2.4, those that rely on cable softening, namely methods No. 1 and No. 3 for the linear spring model, also caused inaccuracies in tension because they changed the total stiffness of the cable. Fig. 17 and Fig. 18 show the effect of this variation on cable tension when performing Manoeuvre II with winch frequency $\omega_{w}=0.3 \mathrm{rad} / \mathrm{s}$, for moderately stiff and highly stiff cable, that is, $E A=10^{7} \mathrm{~N}$ and $E A=10^{8} \mathrm{~N}$.

In spite of the change introduced in cable stiffness, softening the cable segments that were retracted only caused the tension amplitude to decrease about $15 \%$ with respect to the values evaluated with the non-softened cable. This divergence can be considered acceptable in most practical fishing applications. For highly stiff cables, the effect of the softening strategy is even less noticeable and the error in amplitude remains below $5 \%$. 


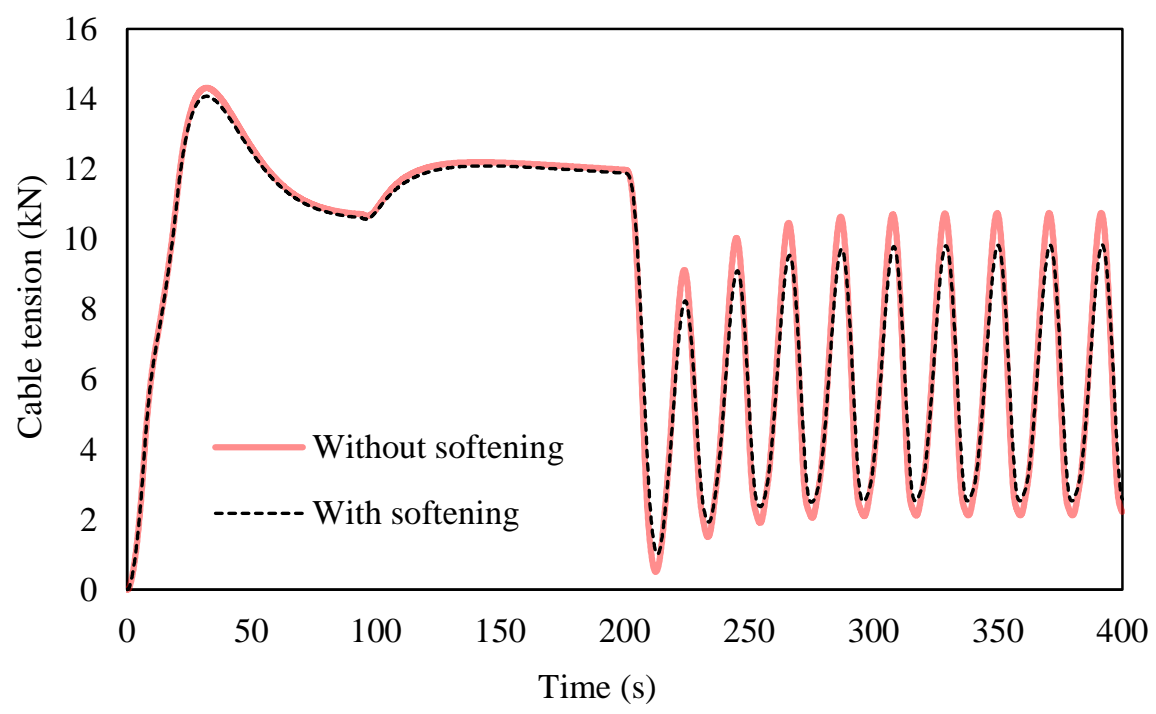

Figure 17: Cable tension during Manoeuvre II $\left(\omega_{w}=0.3 \mathrm{rad} / \mathrm{s}\right)$, obtained with the linear spring approach, $E A=10^{7} \mathrm{~N}$, with and without cable softening

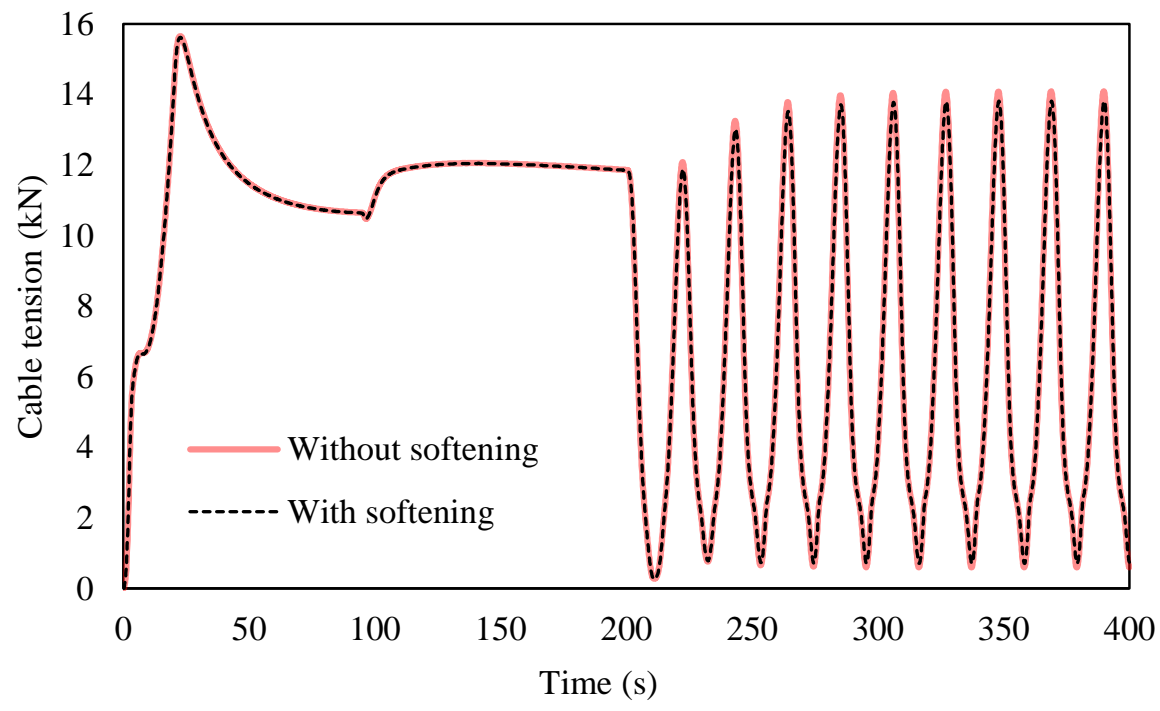

Figure 18: Cable tension during Manoeuvre II $\left(\omega_{w}=0.3 \mathrm{rad} / \mathrm{s}\right)$, obtained with the linear spring approach for highly stiff cable, $E A=10^{8} \mathrm{~N}$, with and without cable softening

\section{Conclusions}

Employing appropriate numerical improvements, real-time simulation of let-out and reel-in manoeuvres with submerged cables can be achieved using both linear-spring and multibody cable models comprising up to a few thousand variables. The cable models and formulations described 
in this work were assessed with two realistic test problems that involved cable pay-out and reelin operations with towed fishing gears. The numerical experiments included towing cables with different values of axial stiffness $E A$ : soft $\left(10^{6} \mathrm{~N}\right)$, moderate $\left(10^{7} \mathrm{~N}\right)$ and stiff $\left(10^{8} \mathrm{~N}\right)$. Each modelling approach has its advantages and limitations and these must be considered when selecting one or another for a given application.

The proposed methods to improve the computational efficiency of reel-in simulation in spring-based cable models, summarized in Table 1, can speed up the simulation by two orders of magnitude. The speed-up became more significant with higher cable axial stiffness. The most efficient technique was method No. 3, i.e., softening the wound cable elements while keeping their mass and stiffness constant. This method achieved real-time performance in most cases. However, method No 3 introduces changes in the total cable stiffness, and hence it may underestimate the cable tension in fast pay-out and reel-in operations with soft and moderately stiff cables. In such scenarios, method No. 2 should be used if a very accurate prediction of cable tension is required. This method only speeds up the simulation by one order of magnitude, and hence it cannot achieve real-time performance in problems that involve fine discretizations or stiff cables.

Concerning the multibody model, the proposed modelling approach based on natural coordinates lead to real-time performance regardless of the formulation used to solve the dynamics. Its excellent computational performance is due to its advantages over other coordinate choices previously used in the literature: lack of explicit parametrization of the rotations, constant mass matrix and absence of Coriolis and velocity-dependent force terms. The AL+FWE formulation was the most efficient one in all cases. The AH+FEW formulation was a 30\% slower, and the two other tested formulations, ALi3p and AL+TR, were between two and four times slower, depending on the problem.

The fastest multibody formulations were always faster than the linear spring methods. Their advantage increased with the cable axial stiffness and the number of variables of the problem. For problems with very low cable stiffness and coarse discretization (100 variables), multibody methods were a $20 \%$ faster. In problems with high cable stiffness and fine discretization (3000 variables), multibody methods were five times faster. However, multibody methods overestimated the cable tension in problems were the cable was subjected to large axial strains. In our numerical experiments, this effect was noticeable when the cable tension exceeded $E A \cdot 10^{-3}$. The effect increased when the winch switched between let-out and reel-in at fast rates, about 


\section{$0.3 \mathrm{rad} / \mathrm{s}$.}

Choosing between multibody and linear spring models can also depend on the implementation environment in which they are used. The linear spring model is simpler to implement and also easy to combine with general ODE solvers, e.g., solvers for lumped-mass models of nets. On the other hand, multibody models lend themselves to joint simulation with other rigid body elements and assemblies, such as realistic models of the doors, anchors, and selective devices. If necessary, both models could be combined by means of co-simulation.

Further improvements to both methods are possible and currently constitute open lines of research. Inaccuracies in cable tension stemming from the infinite axial stiffness of the multibody models can be mitigated using flexible multibody elements to model the cable. Also, variablelength cables are particularly suitable for simulation with recursive, topological formulations for multibody dynamics. A comparison between these methods and the global formulations used in this research is necessary to determine whether they can actually reduce the computational burden of the simulations. Finally, both approaches can benefit from the use of parallelization techniques [19]. Parallel linear solvers could help reduce the elapsed time in the solution of the systems of equations required by multibody simulation. Moreover, parallel implementations allow to integrate each cable separately in fishing assemblies with multiple cables.

\section{Acknowledgements}

The first author would like to acknowledge the support of the Spanish Ministry of Economy through its post-doctoral research program Juan de la Cierva, contract No. JCI-2012-12376.

\section{References}

[1] Bauchau, O.A.: Flexible Multibody Dynamics. Springer (2011). DOI 10.1007/ 978-94-007-0335-3

[2] Baumgarte, J.: Stabilization of constraints and integrals of motion in dynamical systems. Computer Methods in Applied Mechanics and Engineering 1(1), 1-16 (1972). DOI 10. 
1016/0045-7825(72)90018-7

[3] Bayo, E., Avello, A.: Singularity-free augmented Lagrangian algorithms for constrained multibody dynamics. Nonlinear Dynamics 5(2), 209-231 (1994). DOI 10.1007/ BF00045677

[4] Bayo, E., García de Jalón, J., Serna, M.A.: A modified Lagrangian formulation for the dynamic analysis of constrained mechanical systems. Computer Methods in Applied Mechanics and Engineering 71(2), 183-195 (1988). DOI 10.1016/0045-7825(88)90085-0

[5] Bayo, E., Ledesma, R.: Augmented Lagrangian and mass-orthogonal projection methods for constrained multibody dynamics. Nonlinear Dynamics 9(1-2), 113-130 (1996). DOI 10.1007/BF01833296

[6] Bi, C.W., Zhao, Y.P., Dong, G.H., Xu, T.J., Gui, F.K.: Numerical simulation of the interaction between flow and flexible nets. Journal of Fluids and Structures 45, 180-201 (2014). DOI 10.1016/j.jfluidstructs.2013.11.015

[7] Buckham, B., Driscoll, F.R., Nahon, M.: Development of a finite element cable model for use in low-tension dynamics simulation. Journal of Applied Mechanics 71(4), 476-485 (2004). DOI 10.1115/1.1755691

[8] Buckham, B., Nahon, M., Seto, M., Zhao, X., Lambert, C.: Dynamics and control of a towed underwater vehicle system, part I: model development. Ocean Engineering 30(4), 453470 (2003). DOI 10.1016/S0029-8018(02)00029-X

[9] Chen, Y., Davis, T.A., Hager, W.W., Rajamanickam, S.: Algorithm 887: CHOLMOD, supernodal sparse Cholesky factorization and update/downdate. ACM Transactions on Mathematical Software 35(3), art. 22 (2008). DOI 10.1145/1391989.1391995

[10] Cuadrado, J., Cardenal, J., Morer, P., Bayo, E.: Intelligent simulation of multibody dynamics: Space-state and descriptor methods in sequential and parallel computing environments. Multibody System Dynamics 4(1), 55-73 (2000). DOI 10.1023/A:1009824327480

[11] Dopico, D., González, F., Cuadrado, J., Kövecses, J.: Determination of holonomic and nonholonomic constraint reactions in an index-3 augmented Lagrangian formulation with velocity and acceleration projections. Journal of Computational and Nonlinear Dynamics 9(4), paper 041,006 (2014). DOI 10.1115/1.4027671 
[12] Dopico, D., Luaces, A., González, M., Cuadrado, J.: Dealing with multiple contacts in a human-in-the-loop application. Multibody System Dynamics 25(2), 167-183 (2011). DOI 10.1007/s11044-010-9230-y

[13] Eurored: Eurored Vigo S. L. (2014). URL http://euroredvigo.com/es/. (accessed 07.09.2016)

[14] Fritzkowski, P., Kamiński, H.: Dynamics of a rope modeled as a discrete system with extensible members. Computational Mechanics 44(4), 473-480 (2009). DOI 10.1007/ s00466-009-0387-2

[15] Fritzkowski, P., Kamiński, H.: Dynamics of a rope modeled as a multi-body system with elastic joints. Computational Mechanics 46(6), 901-909 (2010). DOI 10.1007/ s00466-010-0524-y

[16] Gansel, L.C., Jensen, Ø., Lien, E., Endresen, P.C.: Forces on nets with bending stiffness: An experimental study on the effects of flow speed and angle of attack. In: Proceedings of the ASME 2012 31st International Conference on Offshore Mechanics and Arctic Engineering OMAE2012, vol. 7, pp. 69-76. Rio de Janeiro, Brazil (2012). DOI 10.1115/OMAE2012-83435

[17] Gobat, J.I., Grosenbaugh, M.A.: Time-domain numerical simulation of ocean cable structures. Ocean Engineering 33(10), 1373-1400 (2006). DOI 10.1016/j.oceaneng.2005.07. 012

[18] González, F., Dopico, D., Pastorino, R., Cuadrado, J.: Behaviour of augmented Lagrangian and Hamiltonian methods for multibody dynamics in the proximity of singular configurations. Nonlinear Dynamics 85(3), 1491-1508 (2016). DOI 10.1007/s11071-016-2774-5

[19] González, F., Luaces, A., Lugrís, U., González, M.: Non-intrusive parallelization of multibody system dynamic simulations. Computational Mechanics 44(4), 493-504 (2009). DOI 10.1007/s00466-009-0386-3

[20] González, M., Dopico, D., Lugrís, U., Cuadrado, J.: A benchmarking system for MBS simulation software: Problem standardization and performance measurement. Multibody System Dynamics 16(2), 179-190 (2006). DOI DOI10.1007/s11044-006-9020-8 
[21] González, M., González, F., Dopico, D., Luaces, A.: On the effect of linear algebra implementations in real-time multibody system dynamics. Computational Mechanics 41(4), 607-615 (2008). DOI 10.1007/s00466-007-0218-2

[22] García de Jalón, J., Bayo, E.: Kinematic and Dynamic Simulation of Multibody Systems. The Real-Time Challenge. Springer-Verlag (1994)

[23] Johansen, V.: Modelling of flexible slender systems for real-time simulation and control applications. Ph.D. thesis, Norwegian University of Science and Technology (2007)

[24] Kamman, J.W., Huston, R.L.: Multibody dynamics modeling of variable length cable systems. Multibody System Dynamics 5(3), 211-221 (2001). DOI 10.1023/A: 1011489801339

[25] Khaled, R., Priour, D., Billard, J.Y.: Numerical optimization of trawl energy efficiency taking into account fish distribution. Ocean Engineering 54, 34-45 (2012). DOI 10.1016/j. oceaneng.2012.07.014

[26] Khaled, R., Priour, D., Billard, J.Y.: Cable length optimization for trawl fuel consumption reduction. Ocean Engineering 58, 167-179 (2013). DOI 10.1016/j.oceaneng.2012.10.001

[27] Kim, K.W., Lee, J.W., Yoo, W.S.: Verification of simulation for unwinding motion of cable in water by physical experiment. Nonlinear Dynamics 77(3), 553-568 (2014). DOI 10.1007/s11071-014-1317-1

[28] Le Dret, H., Lewandowski, R., Priour, D., Chagneau, F.: Numerical simulation of a cod end net - Part 1: Equilibrium in a uniform flow. Journal of Elasticity 76(2), 139-162 (2004). DOI 10.1007/s10659-004-6668-2

[29] Lee, C.W., Lee, J.H., Cha, B.J., Kim, H.Y., Lee, J.H.: Physical modeling for underwater flexible systems dynamic simulation. Ocean Engineering 32(3-4), 331-347 (2005). DOI 10.1016/j.oceaneng.2004.08.007

[30] Lee, C.W., Lee, M.W., Lee, G.H., Yoo, K.Y., Choe, M.Y.: Proceedings of the 11th International Congress of the International Maritime Association of the Mediterranean, chap. Design and performance of a software based fishing simulator, pp. 1245-1250. Taylor \& Francis (2006). DOI 10.1201/9781439833728.ch152 
[31] Lee, J.H., Lee, C.W., Choe, M.Y., Lee, G.H.: Applying fishing-gear simulation software to better estimate fished space as fishing effort. Fisheries and aquatic sciences 14(2), 138147 (2011). DOI 10.5657/FAS.2011.0138

[32] Lee, J.W., Kim, K.W., Kim, H.R., Yoo, W.S.: Prediction of unwinding behaviors and problems of cables from inner-winding spool dispensers. Nonlinear Dynamics 67(3), 17911809 (2012). DOI 10.1007/s11071-011-0106-3

[33] Li, Y.C., Zhao, Y.P., Gui, F.K., Teng, B.: Numerical simulation of the hydrodynamic behaviour of submerged plane nets in current. Ocean Engineering 33(17-18), 2352-2368 (2006). DOI 10.1016/j.oceaneng.2005.11.013

[34] Madsen, N.A.H., Aarsæther, K.G., Herrmann, B., Hansen, K., Jensen, J.H.: The physical behaviour of seine ropes for evaluating demersal seine fishing. In: Proceedings of the ASME 2015 34th International Conference on Ocean, Offshore and Arctic Engineering OMAE2015, OMAE2015-41892. Saint John's, Newfoundland, Canada (2015). DOI 10. 1115/OMAE2015-41892

[35] Moe, H., Fredheim, A., Heide, M.: New net cage designs to prevent tearing during handling. In: Proceedings of the International Congress of International Maritime Association of the Mediterranean, IMAM 2005. Lisbon, Portugal (2005)

[36] Moe, H., Olsen, A., Hopperstad, O.S., Jensen, Ø., Fredheim, A.: Tensile properties for netting materials used in aquaculture net cages. Aquacultural Engineering 37(3), 252-265 (2007). DOI 10.1016/j.aquaeng.2007.08.001

[37] Morgère: Morgère Panneaux de Chalut (2014). URL http://www.morgere.com/en/ products/trawl-doors/item/of .html. (accessed 07.09.2016)

[38] Nédélec, C., Prado, J.: Definition and classification of fishing gear categories. Fisheries Technical Paper No. 222 - Rev. 1. Food and Agriculture Organization of the United Nations, Rome (1990). URL http://www.fao.org/docrep/008/t0367t/t0367t00.HTM

[39] Newmark, N.M.: A method of computation for structural dynamics. Journal of the Engineering Mechanics Division, ASCE 85(EM3), 67-94 (1959)

[40] O'Neill, F.G.: Differential equations governing the geometry of a diamond mesh cod-end of a trawl net. Journal of Applied Mechanics 64(1), 7-14 (1997). DOI 10.1115/1.2787297 
[41] Park, H.I., Jung, D.H., Koterayama, W.: A numerical and experimental study on dynamics of a towed low tension cable. Applied Ocean Research 25(5), 289-299 (2003). DOI 10.1016/j.apor.2004.02.003

[42] Pashen, M., Ritcher, U., Köpnik, W.: Trapese trawl penetration in the seabed. Final report ISBN 3-86009-185-9, University of Rostock (2000). Contract No. 96-006

[43] de la Prada, A., González, M.: Assessing the suitability of gradient-based energy minimization methods to calculate the equilibrium shape of netting structures. Computers \& Structures 135, 128-140 (2014). DOI 10.1016/j.compstruc.2014.01.021

[44] de la Prada, A., González, M.: Quantifying mesh resistance to opening of netting panels: experimental method, regression models, and parameter estimation strategies. ICES Journal of Marine Science 72(2), 697-707 (2015). DOI 10.1093/icesjms/fsu125

[45] de la Prada, A., González, M.: Nonlinear stiffness models of a net twine to describe mesh resistance to opening of flexible net structures. Proceedings of the Institution of Mechanical Engineers, Part M: Journal of Engineering for the Maritime Environment 230(1), 33-44 (2016). DOI 10.1177/1475090214530876

[46] de la Prada, A., Priour, D.: The effect of the bottom boundary layer on trawl behaviour. Ocean Engineering 101, 142-151 (2015). DOI 10.1016/j.oceaneng.2015.04.027

[47] Priour, D.: Calculation of net shapes by the finite element method with triangular elements. Communications in Numerical Methods in Engineering 15(10), 755-763 (1999). DOI 10.1002/(SICI)1099-0887(199910)15:10/755::AID-CNM299〉3.0.CO;2-M

[48] Priour, D.: Numerical optimisation of trawls design to improve their energy efficiency. Fisheries Research 98(1-3), 40-50 (2009). DOI 10.1016/j.fishres.2009.03.015

[49] Priour, D.: A Finite Element Method for Netting. Application to Fish Cages and Fishing Gear. SpringerBriefs in Environmental Science. Springer (2013). DOI 10.1007/ 978-94-007-6844-4

[50] Priour, D., de la Prada, A.: An experimental/numerical study of the catch weight influence on trawl behavior. Ocean Engineering 94, 94-102 (2015). DOI 10.1016/j.oceaneng.2014. 11.016

[51] Reite, K.J.: Modelling and control of trawl systems. Ph.D. thesis, Norwegian University of Science and Technology (2006) 
[52] Sala, A., Lucchetti, A., Buglioni, G.: The change in physical properties of some nylon (PA) netting samples before and after use. Fisheries Research 69(2), 181-188 (2004). DOI 10.1016/j.fishres.2004.05.005

[53] Sala., A., O’Neill, F.G., Buglioni, G., Lucchetti, A., Palumbo, V., Fryer, R.J.: Experimental method for quantifying resistance to the opening of netting panels. ICES Journal of Marine Science 64(8), 1573-1578 (2007). DOI 10.1093/icesjms/fsm113

[54] Servin, M., Lacoursière, C.: Rigid body cable for virtual environments. IEEE Transactions on Visualization and Computer Graphics 14(4), 783-796 (2008). DOI 10.1109/TVCG. 2007.70629

[55] Shimizu, T., Takagi, T., Suzuki, K., Hiraishi, T., Yamamoto, K.: Refined calculation model for NaLA, a fishing net shape simulator, applicable to gill nets. Fisheries Science 70(3), 401-411 (2004). DOI 10.1111/j.1444-2906.2004.00819.x

[56] Takagi, T., Shimizu, T., Korte, H.: Evaluating the impact of gillnet ghost fishing using a computational analysis of the geometry of fishing gear. ICES Journal of Marine Science 64(8), 1517-1524 (2007). DOI 10.1093/icesjms/fsm097

[57] Takagi, T., Shimizu, T., Suzuki, K., Hiraishi, T., Yamamoto, K.: Validity and layout of "NaLA": a net configuration and loading analysis system. Fisheries Research 66(2-3), 235-243 (2004). DOI 10.1016/S0165-7836(03)00204-2

[58] Watson, R., Hoshino, E., Beblow, J., Revenga, C., Kura, Y., Kitchingman, A.: Fishing gear associated with global marine catches. Tech. Rep. 12-6, Fisheries Centre, University of British Columbia, Vancouver (BC), Canada (2004). URL http://www.ecomarres.com/ downloads/12-6.pdf

[59] Yao, Y., Chen, Y., Zhou, H., Yang, H.: A method for improving the simulation efficiency of trawl based on simulation stability criterion. Ocean Engineering 117, 63-77 (2016). DOI 10.1016/j.oceaneng.2016.03.031

[60] Zhu, X., Yoo, W.S.: Suggested new element reference frame for dynamic analysis of marine cables. Nonlinear Dynamics Online first (2016). DOI 10.1007/s11071-016-3055-z 